Homology, Homotopy and Applications, vol.13国, 2011, pp.129-157

\title{
THE FUNDAMENTAL 2-CROSSED COMPLEX OF A REDUCED CW-COMPLEX
}

\author{
JOÃO FARIA MARTINS \\ (communicated by Ronald Brown)
}

\begin{abstract}
We define the fundamental 2-crossed complex $\Omega^{\infty}(X)$ of a reduced CW-complex $X$ from Ellis' fundamental squared complex $\rho^{\infty}(X)$ thereby proving that $\Omega^{\infty}(X)$ is totally free on the set of cells of $X$. This fundamental 2-crossed complex has very good properties with regard to the geometrical realisation of 2-crossed complex morphisms. After carefully discussing the homotopy theory of totally free 2-crossed complexes, we use $\Omega^{\infty}(X)$ to give a new proof that the homotopy category of pointed 3-types is equivalent to the homotopy category of 2 -crossed modules of groups. We obtain very similar results to the ones given by Baues in the similar context of quadratic modules and quadratic chain complexes.
\end{abstract}

\section{Introduction}

A CW-complex $X$ will be called reduced if it has a unique 0-cell, taken to be its basepoint. Let $n$ be a positive integer. An $n$-type is a reduced CW-complex $X$, such that $\pi_{i}(X)$ is trivial for $i>n$. The category \{n-types $\}$ of $n$-types is defined as the category with objects the $n$-types and morphisms the pointed homotopy classes of pointed maps.

It is well known that the fundamental group functor yields an equivalence of categories between the category of groups and the category of 1-types, and that the fundamental crossed module of a $\mathrm{CW}$-complex provides an equivalence of categories between the category of 2-types and the localisation $\mathrm{Ho}(\mathbf{X}$ mod), with respect to weak equivalences, of the model category Xmod of crossed modules; see [16, 20]. Note that the category of crossed modules is equivalent to the category of simplicial groups with Moore complex of length one; for a proof see [14, 20, 23].

The 2-crossed modules were initially defined by Conduché in [14], who proved that the category $\mathbf{2 X m o d}$ of 2 -crossed modules is equivalent to the category of simplicial

This work was supported by CMA/FCT/UNL, under the project PEst-OE/MAT/UI0297/2011. I have benefited from discussions with Björn Gohla.

Received July 31, 2010, revised February 1, 2011, May 4, 2011; published on October 26, 2011. 2000 Mathematics Subject Classification: 55U35; 18D05 18D20, 55Q05, 55 Q15.

Key words and phrases: crossed module, crossed square, squared complex, 2-crossed module, 2crossed complex, quadratic module, 3-type, Gray enriched category.

Article available at http://intlpress.com/HHA/v13/n2/a9 and doi:10.4310/HHA.2011.v13.n2.a9

Copyright (C) 2011, International Press. Permission to copy for private use granted. 
groups with Moore complex of length two. A natural generalisation of a 2-crossed module is a 2-crossed complex [23], obtained from a 2-crossed module by adding a tail of $\mathbb{Z}\left(\pi_{1}\right)$-modules to the underlying complex of a 2-crossed module, in the same way a crossed complex is obtained from a crossed module.

In this article we give a new proof that the category of 3 -types is equivalent to the homotopy category of 2-crossed modules of groups [14]; see also [3, 4, 23]. To this end (and certainly of independent interest), we define the fundamental 2-crossed complex $\Omega^{\infty}(X)$ of a CW-complex $X$, which has very similar properties to Baues' fundamental quadratic chain complex $Q(X)$ of a CW-complex, constructed in [3]. The category of quadratic chain complexes is a reflexive subcategory of the category of 2-crossed complexes (a reflection functor was defined in [2]), and (as observed in [17]) Baues's $Q(X)$ is obtained from $\Omega^{\infty}(X)$ by considering this reflection.

We will often impose a minor technical assumption, also appearing in [17], which is to consider only canonical $\mathrm{CW}$-complexes, where a $\mathrm{CW}$-complex $X$ is canonical if it has a unique 0-cell, all 2-cells attach along pointed maps and the attaching maps of all 3-cells are triad maps. This ensures that the fundamental 2-crossed complex of $X$ may be addressed in a combinatorial way. Nevertheless, the fundamental 2-crossed complex functor is also defined for CW-complexes satisfying only the first and second conditions of the definition of a canonical CW-complex, retaining most properties.

Given a canonical CW-complex $X$, we define $\Omega^{\infty}(X)$ from the fundamental squared complex $\rho^{\infty}(X)$, constructed in [17], after proving that the category of totally free squared complexes (with a chosen basis) and a certain type of maps (neat maps) is isomorphic to the category of totally free 2-crossed complexes, which is not immediate. From Brown-Loday's theorem 11., 12, the fundamental squared complex of a canonical CW-complex is totally free on its cells. In particular, from this construction, we automatically have that $\Omega^{\infty}(X)$ is totally free on the set of cells of $X$, a result which would be difficult to show directly. The combinatorial definition of totally free 2-crossed complexes 21 will, in particular, give a presentation of the first three homotopy groups of a canonical CW-complex, as well as the Whitehead products $\pi_{2} \times \pi_{2} \rightarrow \pi_{3}$, by generators and relations. This appears in [7, 17 in the language of crossed squares, and it is a variant of the presentation considered in [3] in the context of quadratic modules.

We prove, following and using the results of [17], that $\Omega^{\infty}(X)$ has very analogous properties to Baues' fundamental quadratic chain complex of a reduced $\mathrm{CW}$-complex. Apart from the already mentioned fact that $\Omega^{\infty}(X)$ is totally free, this fundamental 2-crossed complex functor has very good properties with regard to the geometric realisation of 2-crossed complex maps and their homotopies. For instance, all 2-crossed complex maps $\Omega^{\infty}(X) \rightarrow \Omega^{\infty}(Y)$ between 3-dimensional canonical CW-complexes can be realised by $\mathrm{CW}$-complex maps $X \rightarrow Y$. This is essentially due to BrownLoday's theorem [11, 12].

We carefully discuss the homotopy theory of totally free 2-crossed complexes, in an analogous way as to what was presented in [3, 25], for quadratic chain complexes and crossed complexes and give detailed calculations.

The fundamental 2-crossed module $\Omega_{3}(X)$ of a CW-complex $X$ can be defined in a similar way to the fundamental 2-crossed complex $\Omega^{\infty}(X)$. All maps $\Omega_{3}(X) \rightarrow \Omega_{3}(Y)$ between the fundamental 2-crossed modules of any two canonical CW-complexes $X$ and $Y$, with $Y$ a 3-type, can be geometrically realised. From this it follows, almost 
immediately, that the category of (pointed) 3-types is equivalent to the homotopy category of 2-crossed modules.

\section{Preliminaries}

Remark 1.1 (Convention on semidirect products). Let $G$ and $E$ be groups. Given a left action $\triangleright$ of $G$ on $E$ by automorphisms, the conventions for the semidirect products $G \ltimes E$ and $E \rtimes G$ are $(g, e)\left(g^{\prime}, e^{\prime}\right)=\left(g g^{\prime},\left(g^{\prime-1} \triangleright e\right) e^{\prime}\right)$ and $(e, g)\left(e^{\prime}, g^{\prime}\right)=$ $\left(e g \triangleright e^{\prime}, g g^{\prime}\right)$, respectively, where $g, g^{\prime} \in G$ and $e, e^{\prime} \in E$.

\subsection{Crossed modules and Peiffer commutators}

A pre-crossed module consists of a group morphism $\partial: E \rightarrow G$ together with a left action $\triangleright$ of $G$ on $E$ by automorphisms such that

$$
\partial(g \triangleright x)=g x g^{-1}, \text { for each } g \in G \text { and each } x \in E .
$$

A crossed module is a pre-crossed module satisfying also the condition

$$
\partial(x) \triangleright y=x y x^{-1} \text {, for each } x, y \in E .
$$

Thus, in a crossed module $(\partial: E \rightarrow G, \triangleright)$, the subgroup $\operatorname{ker}(\partial) \subset E$ is central in $E$.

Let $(\partial: E \rightarrow G, \triangleright)$ be a pre-crossed module. Given $x, y \in E$, their Peiffer commutator is given by

$$
\langle x, y\rangle=\left(x y x^{-1}\right)\left(\partial(x) \triangleright y^{-1}\right) .
$$

A pre-crossed module is a crossed module if and only if all Peiffer commutators vanish.

A morphism $(\psi, \phi)$ between the pre-crossed modules $(\partial: E \rightarrow G, \triangleright)$ and $\left(\partial^{\prime}: E^{\prime} \rightarrow\right.$ $\left.G^{\prime}, \triangleright^{\prime}\right)$ is given by a pair of group morphisms $\psi: E \rightarrow E^{\prime}$ and $\phi: G \rightarrow G^{\prime}$ such that $\phi \circ \partial=\partial^{\prime} \circ \psi$, and such that $\psi(g \triangleright e)=\phi(g) \triangleright^{\prime} \psi(e)$, for each $e \in E$ and $g \in G$. Morphisms of crossed modules are defined analogously, thus the category of crossed modules Xmod is a full subcategory of the category preXmod of pre-crossed modules.

1.1.1. Free pre-crossed modules and crossed modules

Let $G$ be a group and $K$ be a set with a map $\partial_{0}: K \rightarrow G$. We say that a pre-crossed module $(\partial: E \rightarrow G, \triangleright)$ is free on $\partial_{0}$ if there exists a map $i: K \rightarrow E$ with $\partial_{0}=\partial \circ i$ such that the following universal property holds: for any pre-crossed module ( $\partial^{\prime}: E^{\prime} \rightarrow$ $\left.G, \triangleright^{\prime}\right)$ and any map $\psi_{0}: K \rightarrow E^{\prime}$ such that $\partial^{\prime} \circ \psi_{0}=\partial_{0}$, there exists a unique group morphism $\psi: E \rightarrow E^{\prime}$ such that the following diagram commutes:



with ( $\phi$,id) being a morphism of pre-crossed modules. Free crossed modules $(E \rightarrow$ $G, \triangleright)$ are defined analogously, with the only change being that $\left(E^{\prime} \rightarrow G, \triangleright^{\prime}\right)$ is to be 


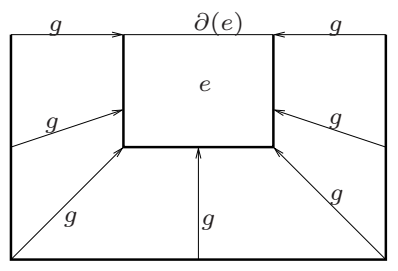

Figure 1: The action of an element $g \in \pi_{1}(Y)$ on an $e \in \pi_{2}(X, Y)$.

a crossed module. Note that the inclusion $\mathbf{X m o d} \rightarrow$ preXmod is full.

The free pre-crossed module $F\left(K \stackrel{\partial_{0}}{\longrightarrow} G\right)$ on a set map $\partial: K \rightarrow G$ is clearly unique up to isomorphism. A model for it is given by considering the free group $F$ on the symbols $(g, k)$ with $g \in G$ and $k \in K$, with action $g \triangleright(h, k)=(g h, k)$ on generators and with boundary map given by $\partial(g, k)=g \partial(k) g^{-1}$ on the free group generators of $F$.

Definition 1.2 (Totally free crossed module). We say that a free pre-crossed module (or crossed module) $E \rightarrow G$ is totally free if $G$ is a free group.

1.1.2. The fundamental crossed module of a pair and Whitehead's theorem Let $(X, Y)$ be a pointed pair of path connected spaces. The obvious boundary map $\partial: \pi_{2}(X, Y) \rightarrow \pi_{1}(Y)$ together with the usual action of $\pi_{1}(Y)$ on $\pi_{2}(X, Y)$ (see Fig. (1)) defines a crossed module $\Pi_{2}(X, Y)$. We thus have a functor $\Pi_{2}$ from the category with objects being the pointed pairs of path-connected spaces and morphisms being the pointed homotopy classes of pointed maps of pairs to the category of crossed modules. All of this is due to Whitehead [24, 25. For a modern treatment see [10].

Theorem 1.3 (Whitehead's theorem). If $X$ is obtained from $Y$ by attaching 2-cells, then $\Pi_{2}(X, Y)$ is the free crossed module on the attaching maps (in $\pi_{1}(Y)$ ) of the 2-cells. In particular, if $X=X^{2}$ is a 2-dimensional reduced $\mathrm{CW}$-complex and $Y=X^{1}$ is its 1-skeleton, then $\Pi_{2}\left(X^{2}, X^{1}\right)$ is a totally free crossed module.

The following theorem [3, 25] follows by induction on the $n$-skeleton $X^{n}$ of the CW-complex $X$, made possible by Whitehead's theorem and the fact that $Y^{1}$ is aspherical.

Theorem 1.4. Let $X$ and $Y$ be reduced 2-dimensional $\mathrm{CW}$-complexes. The map $F \mapsto$ $\Pi_{2}(F)$ yields a one-to-one correspondence between pointed homotopy classes of maps $\left(X, X^{1}, X^{0}\right) \rightarrow\left(Y^{2}, Y^{1}, Y^{0}\right)$ and crossed module maps $\Pi_{2}\left(X^{2}, X^{1}\right) \rightarrow \Pi_{2}\left(Y^{2}, Y^{1}\right)$.

\subsection{Crossed squares and squared complexes}

A crossed square [20] (a notion due Guin-Waléry and Loday) is given by a commutative diagram of groups:

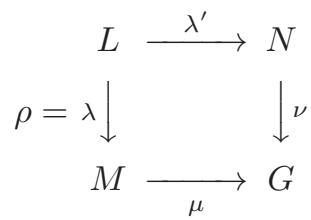


together with left actions of $G$ on $L, M$ and $N$ by automorphisms (denoted by $(g, x) \mapsto$ ${ }^{g} x$, where $g \in G$ and $x$ is in $L, M$ or $N$ ) and a function $h: M \times N \rightarrow L$ (called a crossed pairing), such that

1. All group morphisms are $G$-equivariant, where $G$ acts on itself by conjugation.

2. The morphisms $\lambda, \lambda^{\prime}, \nu, \mu$ and $\nu \circ \lambda^{\prime}=\mu \circ \lambda$ define crossed modules, considering the actions of $N$ and $M$ on $L$ provided by $\nu$ and $\mu$ and the action of $G$ on $L$. Note that $M$ also acts on $N$ through $\mu$, and similarly $N$ acts on $M$ through $\nu$.

3. The crossed pairing $h: M \times N \rightarrow L$ is $G$-equivariant, namely,

$$
h\left({ }^{g} m,{ }^{g} n\right)={ }^{g} h(m, n), \text { for each } m \in M, n \in N \text { and } g \in G .
$$

4. For each $l \in L, m, m^{\prime} \in M$ and $n, n^{\prime} \in N$, we have

$$
\begin{aligned}
h\left(m m^{\prime}, n\right) & =h\left({ }^{m} m^{\prime},{ }^{m} n\right) h(m, n) & h\left(m, n n^{\prime}\right) & =h(m, n) h\left({ }^{n} m,{ }^{n} n^{\prime}\right) \\
\lambda h(m, n) & =m\left({ }^{n} m^{-1}\right) & \lambda^{\prime} h(m, n) & =\left({ }^{m} n\right) n^{-1} \\
h(\lambda l, n) & =l\left({ }^{n} l^{-1}\right) & h\left(m, \lambda^{\prime} l\right) & =\left({ }^{m} l\right) l^{-1} .
\end{aligned}
$$

Morphisms of crossed squares are defined in the obvious way.

A squared complex $\mathcal{A}$ (see $1 \mathbf{1 7}$ ) is given by a commutative diagram of groups:

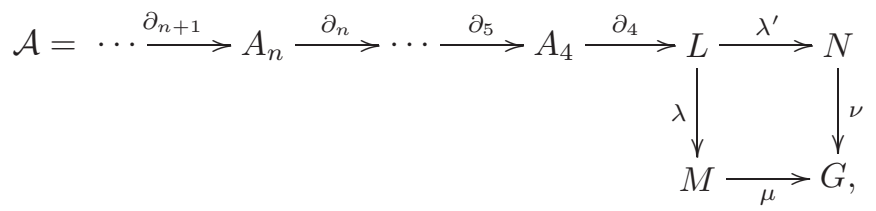

with each $A_{i}$ (where $i \geqslant 4$ ) being abelian, together with an action of $G$ on all the groups, on itself by conjugation, and a $G$-equivariant map $h: M \times N \rightarrow L$, making the square of groups on the right a crossed square, such that

1. All group morphisms are $G$-equivariant.

2. $\partial_{4}\left(A_{4}\right) \subset \operatorname{ker} \lambda \cap \operatorname{ker} \lambda^{\prime}$.

3. Both $\mu(M)$ and $\nu(N)$ act trivially in $A_{n}$ for $n \geqslant 4$.

4. The tail of $\mathcal{A}$, by definition $\left\{A_{n}, \partial_{n} ; n \geqslant 4\right\}$ is a chain complex of abelian groups. If $\pi_{1}(\mathcal{A})$ denotes the cokernel of group map $(n, m) \in N \rtimes M \mapsto \nu(n) \mu(m) \in G$, then there is an induced action of $\pi_{1}(\mathcal{A})$ in each $A_{n}$, where $n \geqslant 4$.

1.2.1. Neat crossed module corners, neat crossed squares and neat squared complexes

A crossed module corner is, by definition, a diagram in the category of groups:

$$
M \stackrel{\mu}{\longrightarrow} G \stackrel{\nu}{\longleftarrow} N,
$$

such that we also have actions of $G$ on $N$ and $M$ by automorphisms, defining two crossed modules. Morphisms of crossed module corners are defined in the obvious way from crossed module morphisms. Given a pre-crossed module $\partial: M \rightarrow P$, consider the following subgroups: $M=\{(m, 1)\}_{m \in M}$ and $\bar{M}=\{\bar{m}\}_{m \in M}=\left\{\left(m, \partial(m)^{-1}\right)\right\}_{m \in M}$ of $M \rtimes P$ (Remark 1.1). Define a pre-crossed module corner $M \longrightarrow M \rtimes P \longleftarrow \bar{M}$, where all group homomorphisms are inclusions and all actions are by conjugation. 
This defines a functor $\iota$ from the category of pre-crossed modules into the category of crossed module corners, which is however not full.

A neat crossed module corner is given by the following data: a pre-crossed module $M \rightarrow P$, a crossed module corner (6), and group isomorphisms $T_{+}: \bar{M} \rightarrow N$ and $T_{0}: M \rtimes P \rightarrow G$, making the following diagram commutative and preserving all actions (that is (id, $T_{0}, T_{+}$) is to be an isomorphism of crossed module corners):



A neat morphism between neat crossed module corners $C$ and $C^{\prime}$, associated to the pre-crossed modules $(M \rightarrow P)$ and $\left(M^{\prime} \rightarrow P^{\prime}\right)$ is a crossed module corner map $C \rightarrow$ $C^{\prime}$ induced, through $\iota$, by a pre-crossed module morphism $(M \rightarrow G) \rightarrow\left(M^{\prime} \rightarrow G^{\prime}\right)$. We therefore have an equivalence of categories between the category of pre-crossed modules and the category of neat crossed module corners and neat morphisms.

Definition 1.5 (Neat squared complex and neat crossed square). A squared complex (5) is said to be neat if the associated crossed module corner $M \stackrel{\mu}{\rightarrow} G \stackrel{\nu}{\leftarrow} N$ is neat. A neat morphism between neat squared complexes is a morphism of squared complexes which is neat at the level of crossed module corners. We analogously define the category of neat crossed squares and neat morphisms.

1.2.2. The fundamental crossed square of a pointed triad

Let $(X ; A, B)$ be a pointed triad. In other words, $X$ is a path-connected pointed space with two path-connected subspaces $A$ and $B$, containing the basepoint, such that the intersection $W=A \cap B$ is path connected. Consider the triad homotopy group $\pi_{3}(X ; A, B)$; see for example [1, 6]. There are obvious morphisms $\pi_{3}(X ; A, B) \rightarrow$ $\pi_{2}(A, W)$ and $\pi_{3}(X ; A, B) \rightarrow \pi_{2}(B, W)$. There also exists an action of $\pi_{1}(W)$ on all of these groups, and all boundary maps are $\pi_{1}(W)$-equivariant. The generalised Whitehead product [1, 5] defines a $\pi_{1}(W)$-equivariant (set) map $h: \pi_{2}(A, W) \times$ $\pi_{2}(B, W) \rightarrow \pi_{3}(X ; A, B)$. It is proved in [17, 18] that given any pointed triad $(X ; A, B)$, the square of groups (with obvious morphisms)



together with the generalised Whitehead product and the actions of $\pi_{1}(W)$, defines a crossed square $\rho_{3}(X ; A, B)$.

1.2.3. Canonical CW-complexes and bifiltered maps Let $B^{2}=\{z \in \mathbb{C}:|z-1| \leqslant 1\}$ and $B_{-}^{2}=\{z \in \mathbb{C}:|z-1 / 2| \leqslant 1 / 2\}$. Let also $B_{+}^{2}=$ $B^{2} \backslash \operatorname{int}\left(B_{-}^{2}\right)$. This yields a CW-decomposition of $B^{2}$ with a 0 -cell at $\{0\}$ (taken as its basepoint), two 1 -cells and two 2 -cells. This also defines a pointed triad $\left(B^{2} ; B_{-}^{2}, B_{+}^{2}\right)$. (We refer to Fig. 2.) We can also define a triad structure on $S^{2}$, taking $S_{-}^{2}$ and $S_{+}^{2}$ to 


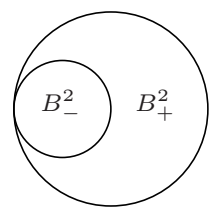

Figure 2: The triad structure $\left(B^{2} ; B_{-}^{2}, B_{+}^{2}\right)$ on the 2-ball $B^{2}$.

be the southern and northern hemispheres. The following definition is due to Ellis; see [17]. As usual $X^{n}$ denotes the $n$-skeleton of the CW-complex $X$.

Definition 1.6 (Canonical CW-complexes). A CW-complex $X$ is canonical if

1. The CW-complex $X$ has a unique 0 -cell, taken as its basepoint.

2. The attaching map $\partial B^{2} \rightarrow X^{1}$ of each 2-cell is pointed. This thus defines an obvious triad $\left(X^{2}, X_{-}^{2}, X_{+}^{2}\right)$ induced from the triad structure in $B^{2}$.

3. Each 3-cell attaches along a pointed triad map $\left(S^{2} ; S_{-}^{2}, S_{+}^{2}\right) \rightarrow\left(X^{2} ; X_{-}^{2}, X_{+}^{2}\right)$.

Definition 1.7 (Bifiltered map). Let $X$ and $Y$ be canonical CW-complexes with their unique 0-cells taken to be the basepoints. A map $f: X \rightarrow Y$ is said to be bifiltered if it is cellular, thus pointed, and moreover $f\left(X_{+}^{2}\right) \subset Y_{+}^{2}$ and $f\left(X_{-}^{2}\right) \subset Y_{-}^{2}$. A homotopy $H$ connecting the bifiltered maps $f, g: X \rightarrow Y$ is bifiltered if it is filtered (i.e., $\left.H\left(X^{i} \times I\right) \subset Y^{i}\right)$ and moreover $H\left(X_{+}^{2} \times I\right) \subset Y_{+}^{2}$ and $H\left(X_{-}^{2} \times I\right) \subset Y_{-}^{2}$.

Triad maps $f:\left(X ; X_{-}^{2}, X_{+}^{2}\right) \rightarrow\left(Y, Y_{-}^{2}, Y_{+}^{2}\right)$ between canonical CW-complexes need not be bifiltered, since bifiltered maps should further satisfy $f\left(X^{1}\right) \subset Y^{1}$.

Theorem 1.8 (Bifiltered approximation theorem). Each map $f: X \rightarrow Y$ between canonical CW-complexes is homotopic to a bifiltered map $f^{\prime}$. If $f$ is bifiltered when restricted to a subcomplex $Z$ of $X$, then such homotopy can be chosen relative to $Z$.

Proof. Choose a cellular approximation $g$ to $f: X \rightarrow Y$ and a homotopy $H_{1}: X \times$ $I \rightarrow Y$ connecting $f$ and $g$ and constant over $Z$. By using the following lemma, there exists a map $H_{2}^{\prime}:\left(Z \cup X^{2}\right) \times I \cup(X \times\{0\}) \rightarrow Y$, stable in $Z$, extending $g$ and connecting the restriction of $g$ to $Z \cup X^{2}$ with a bifiltered map $g^{\prime}: X^{2} \cup Z \rightarrow Y$. By a trivial inductive argument, $H_{2}^{\prime}$ extends to all of $X \times I$, defining a homotopy connecting $g$ and a bifiltered map $f^{\prime}$.

Lemma 1.9. Every cellular map $f: B^{2} \rightarrow Y^{2}$ (where $B^{2}$ has a $\mathrm{CW}$-decomposition with single 0-, 1- and 2-cells) is homotopic to a bifiltered map $f^{\prime}: B^{2} \rightarrow Y^{2}$ through a homotopy which fixes $S^{1}=\partial B^{2}$.

Proof. Let $R$ be made of the left, right and bottom faces of $D^{2}=[0,1]^{2}$. Consider a homeomorphism $r: D^{2} / R \rightarrow B^{2}$. Then the element $f \circ r: D^{2} \rightarrow X$ is an element of $\pi_{2}\left(Y^{2}, Y^{1}\right)$. Any 2-cell of $v_{i}$ of $Y$ defines a map $e_{i}: D^{2} / R \rightarrow Y^{2}$, yielding an element of $\pi_{2}\left(Y^{2}, Y^{1}\right)$, in the same way. As an element of $\pi_{2}\left(Y^{2}, Y^{1}\right)$, the element $f \circ r$ is homotopic to an element of the form

$$
\phi=\left(g_{1} \triangleright e_{i_{1}}\right)^{\theta_{1}}\left(g_{2} \triangleright e_{i_{2}}\right)^{\theta_{2}} \cdots\left(g_{n} \triangleright e_{i_{n}}\right)^{\theta_{n}} \text {, where } g_{i} \in \pi_{1}\left(Y^{1}\right) \text { and } \theta_{i} \in \mathbb{Z},
$$

by using the results of 1.1.1 and 1.1.2, since the crossed module $\Pi_{2}\left(Y^{2}, Y^{1}\right)$ is free. Consider a homotopy in $Y^{1}$ from $\partial(\phi)$ to $\partial(f \circ r)$. Let $\phi^{\prime}$ be the vertical concatenation 
of $\phi$ and this homotopy, defining a map $\phi^{\prime}: D^{2} / R \rightarrow Y$ such that the restriction of it to the top of $D^{2}$ is $\partial(f \circ r)$. Given the form of the action of $\pi_{1}\left(Y^{1}\right)$ on $\pi_{2}\left(Y^{2}, Y^{1}\right)$ (see Fig. 1]) the map $f^{\prime}=\phi^{\prime} \circ r^{-1}: B^{2} \rightarrow Y$ is bifiltered, and by the construction of $f^{\prime}$ there exists a homotopy connecting $f$ and $f^{\prime}$, stable on the boundary $S^{1}$ of $B^{2}$.

We have categories $\mathcal{C W}_{b}$ of canonical CW-complexes and bifiltered maps and $\mathrm{CW}_{b} / \cong$ of canonical $\mathrm{CW}$-complexes and bifiltered maps up to bifiltered homotopy. We will also consider the categories $\mathrm{eW}$ and $\mathrm{eW} / \cong$ of canonical CW-complexes and pointed maps and of canonical CW-complexes and pointed maps up to pointed homotopy. By using the bifiltered approximation theorem (see the construction below), any reduced $\mathrm{CW}$-complex is homotopic to a canonical $\mathrm{CW}$-complex, a fact mentioned in 17. Therefore, the category $\mathrm{eW} / \cong$ is equivalent to the category $\mathbf{C W} / \cong$ of CW-complexes and pointed homotopy classes of pointed maps. Define also a full subcategory $\left\{3\right.$-types ${ }^{\text {can }}$ of the category $\{3$-types $\}$, called the category of canonical 3 -types, with objects being the 3 -types whose underlying CW-complex is canonical. Clearly the inclusion $\{3 \text {-types }\}^{\text {can }} \rightarrow\{3$-types $\}$ is an equivalence of categories.

Consider a CW-complex $X$ satisfying conditions 1 and 2 of the definition of a canonical CW-complex. Let $Y$ be a homotopically equivalent $\mathrm{CW}$-complex, obtained from $X$ by substituting the attaching maps of all 3-cells by homotopic triad maps, whose existence follows from the bifiltered approximation theorem. Note $X^{2}=Y^{2}$.

Lemma 1.10. There exists a triad homotopy equivalence $\left(X, X_{-}^{2}, X_{+}^{2}\right) \rightarrow\left(Y, Y_{-}^{2}, Y_{+}^{2}\right)$ which is the identity over 2 -skeletons, thus in particular defining a bifiltered homotopy equivalence if $X$ and $Y$ are 3-dimensional $\mathrm{CW}$-complexes.

Proof. Clearly there exist homotopy equivalences $f: X \rightarrow Y$ and $g: Y \rightarrow X$ which are the identity over 2-skeletons. Consider the constant homotopies $H_{2}: f^{2} \circ g^{2} \rightarrow$ $\operatorname{id}_{Y^{2}}$ and $K_{2}: g^{2} \circ f^{2} \rightarrow \operatorname{id}_{X^{2}}$, where $f^{2}$ is the restriction of $f$ to the 2-skeleton $X^{2}$ of $X$, and analogously for $g^{2}$. Since the inclusions of $X^{2}$ in $X$ and of $Y^{2}$ in $Y$ are cofibrations, we can find a homotopy inverse $g^{\prime}$ of $f$ extending $g^{2}$ such that there exist homotopies $H: f \circ g^{\prime} \rightarrow \operatorname{id}_{Y}$, extending $H_{2}$, and $K: g^{\prime} \circ f \rightarrow \mathrm{id}_{X}$, extending the following concatenation of homotopies (which is clearly a constant homotopy)

$$
\operatorname{id}_{X^{2}}=g^{2} \circ f^{2} \stackrel{\left(g^{2} f^{2} K_{2}\right)^{-1}}{\longrightarrow} g^{2} \circ f^{2} \circ g^{2} \circ f^{2} \stackrel{g^{2} H_{2} f^{2}}{\longrightarrow} g^{2} \circ f^{2} \stackrel{K_{2}}{\longrightarrow} \operatorname{id}_{X^{2}} .
$$

(We use the notation and the main result of [8, 7.4].) Therefore, $f$ and $g^{\prime}$ define a triad homotopy equivalence, which is the identity over 2-skeletons.

1.2.4. The fundamental crossed square $\rho_{3}(X)$ of a canonical CW-complex Let $X$ be a canonical CW-complex. The fundamental crossed square of it [17] is

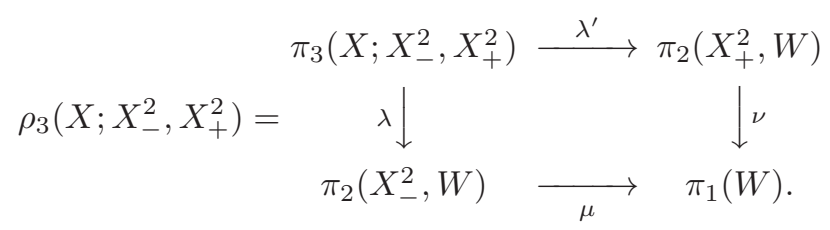

We have two crossed modules, $\Pi_{2}\left(X_{-}^{2}, W\right)$ and $\Pi_{2}\left(X_{+}^{2}, W\right)$, which are both totally free, since $X_{-}^{2}$ and $X_{+}^{2}$ each are obtained from $W=W_{X}=X_{-}^{2} \cap X_{+}^{2}$ (a 1-dimensional 
CW-complex) by attaching 2-cells. Note that $W \cong X^{1} \bigvee \bigvee_{\{2 \text {-cells }\}} S^{1}$, thus $\pi_{1}(W)$ is the free group on the set $A \cup B$, where $A$ and $B$ are the sets of 1- and 2-cells. Given that both spaces $X_{-}^{2}$ and $X_{+}^{2}$ are aspherical, we have two exact sequences:

$$
0 \rightarrow \pi_{2}\left(X_{+}^{2}, W\right) \stackrel{\nu}{\rightarrow} \pi_{1}(W) \stackrel{p_{+}}{\rightarrow} \pi_{1}\left(X_{+}^{2}\right) \cong \pi_{1}\left(X^{1}\right) \rightarrow 1
$$

and

$$
0 \rightarrow \pi_{2}\left(X_{-}^{2}, W\right) \stackrel{\mu}{\rightarrow} \pi_{1}(W) \stackrel{p_{-}}{\longrightarrow} \pi_{1}\left(X_{-}^{2}\right) \cong \pi_{1}\left(X^{1}\right) \rightarrow 1,
$$

which are split given that $\pi_{1}\left(X^{1}\right)$ is a free group $\left(p_{+}\right.$and $p_{-}$are induced by the inclusion of $W$ in $X_{+}^{2}$ and $X_{-}^{2}$, respectively). For the applications below, we enhance the commutative square (17) to the following diagram, with exact mid row and column:

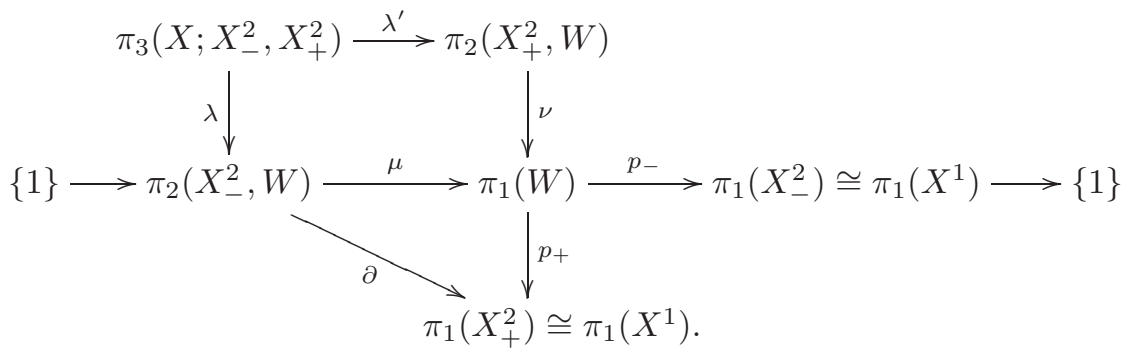

Note that $\nu$ and $\mu$ are injective. The following important lemma is implicit in $\mathbf{1 7}$.

Lemma 1.11. Define a projection map $p_{+}: \pi_{1}(W) \rightarrow \pi_{1}\left(X^{1}\right)=\pi_{1}\left(X_{+}^{2}\right)$ given by the inclusion of $W$ on $X_{+}^{2}$. Consider the inclusion map $\pi_{1}\left(X^{1}\right) \rightarrow \pi_{1}(W)$ given by the inclusion of $X^{1}$ in $W$, thus corresponding to the inclusion of free generators. Define an action of $\pi_{1}\left(X^{1}\right)$ on $\pi_{2}\left(X_{-}^{2}, W\right)$ provided by the action of $\pi_{1}(W)$ on $\pi_{2}\left(X_{-}^{2}, W\right)$ and the inclusion of $\pi_{1}\left(X^{1}\right)$ in $\pi_{1}(W)$. Let $\partial=p_{+} \circ \mu$. Then

1. With the action just defined, $\left(\pi_{2}\left(X_{-}^{2}, W\right) \stackrel{\partial}{\rightarrow} \pi_{1}\left(X^{1}\right)\right)$ is the free pre-crossed module on the attaching maps of the 2 -cells of $X$ in $\pi_{1}\left(X^{1}\right)$.

2. Let $M=\pi_{2}\left(X_{-}^{2}, W\right)$. The exact sequence (8), which is split, gives an isomorphism of crossed modules, where the first crossed module is given by the normal inclusion $m \in M \mapsto(m, 1) \in M \rtimes \pi_{1}\left(X^{1}\right)$ and the action is by conjugation:

$$
\left(M \rightarrow M \rtimes \pi_{1}\left(X^{1}\right)\right) \stackrel{\left(\mathrm{id}, T_{0}\right)}{\longrightarrow} \Pi_{2}\left(X_{-}^{2}, W\right) \doteq\left(\pi_{2}\left(X_{-}^{2}, W\right) \rightarrow \pi_{1}(W)\right) .
$$

Explicitly, $T_{0}(m, g)=\mu(m) g$, where $m \in M$ and $g \in \pi_{1}\left(X^{1}\right)$.

3. Under the identification $\pi_{1}(W) \cong M \rtimes \pi_{1}\left(X^{1}\right)$, the map $p_{+}: \pi_{1}(W) \rightarrow \pi_{1}\left(X^{1}\right)$ is given by

$$
(m, w) \mapsto \partial(m) w .
$$

4. In addition, from $\pi_{2}\left(X_{+}^{2}, W\right)=\operatorname{ker}\left(p_{+}\right)$, it follows that there exists a naturally defined isomorphism

$$
\bar{M}=\{\bar{m}\}_{m \in M} \stackrel{T_{+}}{\longrightarrow} \pi_{2}\left(X_{+}^{2}, W\right)
$$

where $\bar{m}=\left(m, \partial(m)^{-1}\right)$, and moreover, through $\left(T_{+}, T_{0}\right)$, the crossed module $\Pi_{2}\left(X_{+}^{2}, W\right)$ is isomorphic to the one given by the inclusion of $\bar{M}$ in $M \rtimes \pi_{1}\left(X^{1}\right)$. 
In particular, the fundamental crossed square of a CW-complex is neat 1.2.1, and we also have a functor $\rho_{3}$ from the category of canonical CW-complex and bifiltered maps to the category of neat crossed squares and neat maps; see also 1.2.6.

1.2.5. Free and totally free crossed squares and squared complexes

Consider a crossed module corner (6). Suppose we have a set $K$ together with maps $\lambda_{0}: K \rightarrow M$ and $\lambda_{0}^{\prime}: K \rightarrow N$, such that $\nu \circ \lambda_{0}^{\prime}=\mu \circ \lambda_{0}$. A crossed square

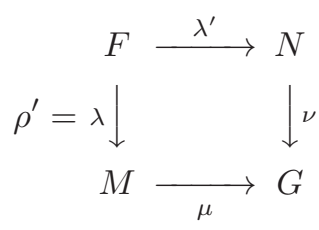

is said to be free on $\left(\lambda_{0}, \lambda_{0}^{\prime}\right)$ if there exists an inclusion map $i: K \rightarrow F$ such that for each crossed square of the form (11) and each map $\phi: K \rightarrow L$ such that $\lambda^{\prime} \circ \phi=\lambda_{0}^{\prime}$ and $\lambda \circ \phi=\lambda_{0}$, there exists a unique crossed square map $\rho^{\prime} \rightarrow \rho$, which is the identity on $M, N$ and $G$, and such that the underlying map $\bar{\phi}: F \rightarrow L$ extends $\phi: K \rightarrow L$. For details see 11, 17, 22].

The following definition is due to Ellis ([17]) and is motivated by Lemma 1.11.

Definition 1.12 (Totally free and 2-free crossed square). Let $S_{2}$ be a set together with a map $\partial_{0}: S_{2} \rightarrow F$, where $F$ is the free group on a set $S_{1}$. Let $(M \stackrel{\partial}{\rightarrow} F)$ be the associated totally free pre-crossed module. Consider the following neat crossed module corner, 1.2.1, with all maps being inclusions:



As above, $\bar{M}=\{\bar{m}\}_{m \in M}=\left\{\left(m, \partial(m)^{-1}\right\}_{m \in M}\right.$. Consider a set-map $\lambda_{0}: K \rightarrow \operatorname{ker}(\partial)$ $\subset M$, and let $\lambda_{0}^{\prime}(k)=\overline{\lambda_{0}(k)}=\left(\lambda_{0}(k), 1\right)$. Then the free crossed square on $\lambda_{0}: K \rightarrow M$ and $\lambda_{0}^{\prime}: K \rightarrow \bar{M}$ is said to be the totally free crossed square on $\left(S_{1}, S_{2}, K, \partial_{0}, \lambda_{0}\right)$. A crossed square whose underlying crossed module corner has the form (9), with $M \rightarrow F$ being a totally free pre-crossed module, will be called 2-free.

Definition 1.13 (Free and 2-free crossed squares with a chosen basis). The category of totally free crossed squares with a chosen basis has objects $\left(S_{1}, S_{2}, K, \partial_{0}, \lambda_{0}\right)$, as above, and morphisms crossed square maps between the associated totally free crossed squares. We analogously define the category of 2 -free crossed squares with a chosen (pre-crossed module) basis.

The following non-trivial theorem is a particular case of the van Kampen theorem for diagrams of spaces, which appears in [11, 12]. Let $X$ be a canonical CW-complex. Let $K$ be the set of its 3-cells, each attaching through a triad map $\left(S^{2} ; S_{-}^{2}, S_{+}^{2}\right) \rightarrow\left(X^{2} ; X_{-}^{2}, X_{+}^{2}\right)$ and therefore defining maps $\lambda_{0}^{\prime}: K \rightarrow \pi_{2}\left(X_{+}^{2}, W\right)$ and $\lambda_{0}: K \rightarrow \pi_{2}\left(X_{-}^{2}, W\right)$. 
Theorem 1.14 (Brown-Loday). The fundamental crossed square of a canonical CWcomplex $X$ of dimension three is the free crossed square on the maps above and is, in particular (by Lemma 1.11), totally free.

If $X$ is a 3 -dimensional $\mathrm{CW}$-complex satisfying conditions 1 and 2 of the definition of a canonical CW-complex, then $X$ is bifiltered homotopic to a canonical CW-complex (Lemma 1.10), and therefore its fundamental crossed square is totally free as well.

A squared complex $\mathcal{A}$ as in (5) is said to be totally free if its associated crossed squared is totally free and also each $A_{n}$ is a free $\mathbb{Z}\left(\pi_{1}(\mathcal{A})\right.$ )-module. There exist categories of totally free squared complexes, with or without chosen basis, 1.2.5. From our previous discussion in 1.2.4 and 1.2.5, it follows that (see $1 \mathbf{1 7}$ ) if $X$ is a canonical $\mathrm{CW}$-complex, then the diagram of groups

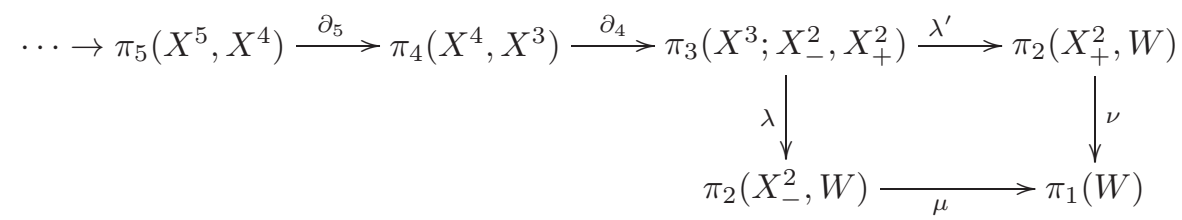

(where $W=X_{-}^{2} \cap X_{+}^{2}$ ) is a squared complex called the fundamental squared complex $\rho^{\infty}(X)$ of $X$, which is totally free on the cells of $X$.

Remark 1.15. A totally free or 2-free squared complex (or crossed square) with a chosen basis is automatically neat.

Consider the categories $\mathrm{eW}_{b}$ and $\mathrm{eW}_{b} / \cong$, of canonical CW-complexes and bifiltered maps, up to bifiltered homotopy in the latter case; see 1.2.3. We have functors $\rho^{\infty}$ from $\mathcal{C} \mathcal{W}_{b}$ and $\mathcal{C} \mathcal{W}_{b} / \cong$ into the category of neat squared complexes and neat maps. These functors can also be seen as taking values in the category of totally free squared complexes with a chosen basis and neat maps.

1.2.6. Geometric realisation of squared complex maps

Squared complex maps have nice properties with regard to geometric realisation, as shown in the following result due to Ellis; see [17, Propositions 8 and 9], proved by an obvious induction on $n$-skeletons, noting that for a canonical $\mathrm{CW}$-complex $Y$ the spaces $Y_{ \pm}^{2}$ are aspherical.

Theorem 1.16 (Ellis). We have

- Given a 5-truncated totally free squared complex $\mathcal{F}$, there exists a 5-dimensional canonical $\mathrm{CW}$-complex $X$ with $\rho^{\infty}(X)$ isomorphic to $\mathcal{F}$.

- Given canonical $\mathrm{CW}$-complexes $X$ and $Y$ with $X$ of dimension $\leqslant 4$, any squared complex map $F: \rho^{\infty}(X) \rightarrow \rho^{\infty}(Y)$ can be realised by a pointed map $f: X \rightarrow Y$ with $f\left(X^{i}\right) \subset Y^{i}$, if $i \geqslant 3$ and $f\left(X_{ \pm}^{2}\right) \subset Y_{ \pm}^{2}$.

Remark 1.17. In the second assertion of the previous theorem, note that the realisation map $f: X \rightarrow Y$ need not be bifiltered, for we may need to consider $f\left(X^{1}\right) \not \subset Y^{1}$, but with $f\left(X^{1}\right) \subset Y_{-}^{2} \cap Y_{+}^{2}$. By Ellis' proof we can however see (in the conditions above) that neat squared complex maps $\rho^{\infty}(X) \rightarrow \rho^{\infty}(Y)$ can be realised by bifiltered maps $X \rightarrow Y$. 


\section{2-crossed modules and 2-crossed complexes}

\subsection{Definition of 2-crossed modules}

We follow the conventions of 14, 19 for the definition of a 2-crossed module $[\mathbf{1 4}$.

Definition 2.1. A 2-crossed module is given by a chain complex of groups:

$$
L \stackrel{\delta}{\rightarrow} E \stackrel{\partial}{\rightarrow} G
$$

together with left actions $\triangleright$ by automorphisms of $G$ on $L$ and $E$ (and on $G$ by conjugation) and a $G$-equivariant function $\{\}:, E \times E \rightarrow L$ (called the Peiffer lifting). These are to satisfy:

1. $L \stackrel{\delta}{\rightarrow} E \stackrel{\partial}{\rightarrow} G$ is a chain complex of $G$-modules (in other words $\partial$ and $\delta$ are $G$-equivariant and $\partial \circ \delta=1)$.

2. $\delta(\{e, f\})=\langle e, f\rangle$, for each $e, f \in E$. Recall that $\langle e, f\rangle=\left(e f e^{-1}\right)\left(\partial(e) \triangleright f^{-1}\right)$.

3. $[l, k]=\{\delta(l), \delta(k)\}$, for each $l, k \in L$. Here $[l, k]=l k l^{-1} k^{-1}$.

4. $\{\delta(l), e\}\{e, \delta(l)\}=l\left(\partial(e) \triangleright l^{-1}\right)$, for each $e \in E$ and $l \in L$.

5. $\{e f, g\}=\left\{e, f g f^{-1}\right\} \partial(e) \triangleright\{f, g\}$, for each $e, f, g \in E$.

6. $\{e, f g\}=\{e, f\}(\partial(e) \triangleright f) \triangleright^{\prime}\{e, g\}$, where $e, f, g \in E$.

Here we have put

$$
e \triangleright^{\prime} l=l\left\{\delta(l)^{-1}, e\right\} \text {, where } l \in L \text { and } e \in E .
$$

It follows from the previous axioms that $\triangleright^{\prime}$ is a left action of $E$ on $L$ by automorphisms (see 9, 14]). Together with the map $\delta: L \rightarrow M$, this defines a crossed module, and in particular $\operatorname{ker}(\delta) \subset L$ is central in $L$.

For a proof of the following lemma see [19.

Lemma 2.2. In a 2-crossed module $(L \stackrel{\delta}{\rightarrow} E \stackrel{\partial}{\rightarrow} G, \triangleright,\{\}$,$) we have, for each e, f, g \in$ $E, a \in G$ and $k \in L$,

$$
\begin{aligned}
& \{e f, g\}=\left(e \triangleright^{\prime}\{f, g\}\right)\{e, \partial(f) \triangleright g\}, \quad\{e, f g\}=\left(\left(e f e^{-1}\right) \triangleright^{\prime}\{e, g\}\right)\{e, f\}, \\
& \left\{1_{E}, e\right\}=\left\{e, 1_{E}\right\}=1_{L}, \quad a \triangleright\left(e \triangleright^{\prime} k\right)=(a \triangleright e) \triangleright^{\prime}(a \triangleright k), \\
& \{e, f\}^{-1}=\partial(e) \triangleright\left\{e^{-1}, e f e^{-1}\right\}, \quad\{e, f\}^{-1}=\left(e f e^{-1}\right) \triangleright^{\prime}\left\{e, f^{-1}\right\}, \\
& \{e, f\}^{-1}=(\partial(e) \triangleright f) \triangleright^{\prime}\left\{e, f^{-1}\right\}, \quad\{e, f\}^{-1}=e \triangleright^{\prime}\left\{e^{-1}, \partial(e) \triangleright f\right\} \text {. }
\end{aligned}
$$

Note that

$$
\left\{\delta(l)^{-1}, e\right\}^{-1} l^{-1}=e \triangleright^{\prime} l^{-1}=l^{-1}\{\delta(l), e\} \text { and } \partial(e) \triangleright l=\left(e \triangleright^{\prime} l\right)\left\{e, \partial(l)^{-1}\right\},
$$

thus $\partial(e) \triangleright l=e \triangleright^{\prime} l$ if $e \in E$ and $l \in \operatorname{ker} \delta$; see equation (11). For each $a, b, c \in E$ we have

$$
a \triangleright^{\prime}\{b, c\}=\partial(a) \triangleright\{b, c\}\left\{a,\langle b, c\rangle^{-1}\right\}=\{\partial(a) \triangleright b, \partial(a) \triangleright c\}\left\{a,(\partial(b) \triangleright c) b c^{-1} b^{-1}\right\} .
$$

A morphism between the 2-crossed modules $\left(L_{1} \rightarrow E_{1} \rightarrow G_{1}, \triangleright_{1},\{,\}_{1}\right)$ and $\left(L_{2} \rightarrow\right.$ $\left.E_{2} \rightarrow G_{2}, \triangleright_{2},\{,\}_{2}\right)$ is given by three group morphisms: $\eta: L_{1} \rightarrow L_{2}, \psi: E_{1} \rightarrow E_{2}$ and $\phi: G_{1} \rightarrow G_{2}$, defining a chain map between the underlying complexes, such that

$$
\eta\left(\{e, f\}_{1}\right)=\{\psi(e), \psi(f)\}_{2}, \quad \eta\left(g \triangleright_{1} x\right)=\phi(g) \triangleright_{2} \eta(x) \text { and } \psi\left(g \triangleright_{1} e\right)=\phi(g) \triangleright_{2} \psi(e) .
$$


Example 2.3. Given a pre-crossed module $E \rightarrow G$, we consider the Peiffer subgroup $\langle E, E\rangle$ of $E$, generated by the Peiffer commutators $\langle a, b\rangle$, 1.1.1. Then $\langle E, E\rangle \rightarrow E \rightarrow$ $G$ is a 2 -crossed module, where the Peiffer lifting is $\{a, b\}=\langle a, b\rangle$.

\subsection{An equivalence $\Psi$ between the categories of neat squared complexes and neat maps and 2-crossed complexes}

Definition 2.4. A 2-crossed complex [13, 21] is given by a chain complex of groups

$$
\cdots \stackrel{\partial_{n+1}}{\longrightarrow} A_{n} \stackrel{\partial_{n}}{\longrightarrow} \cdots \stackrel{\partial_{5}}{\longrightarrow} A_{4} \stackrel{\partial_{4}}{\longrightarrow} L \stackrel{\delta}{\longrightarrow} E \stackrel{\partial}{\longrightarrow} G,
$$

together with actions of $G$ on all the groups of the complex (and on itself by conjugation) and a $G$-equivariant map $\{\}:, E \times E \rightarrow L$ such that:

1. $L \stackrel{\delta}{\longrightarrow} E \stackrel{\partial}{\longrightarrow} G$ is a 2-crossed module.

2. All groups $A_{n}$ where $n \geqslant 4$ are abelian.

3. The action of $\partial(E) \subset G$ on $A_{n}$ where $n \geqslant 4$ is trivial.

The tail of a 2-crossed complex is, by definition, the complex $\left\{A_{n}, n \geqslant 4\right\}$ of abelian groups. This is analogous to the squared complex case.

Going back to crossed squares, suppose we have a pre-crossed module $(M \rightarrow G, \triangleright)$. Consider a neat crossed square 1.2.1 of the form:

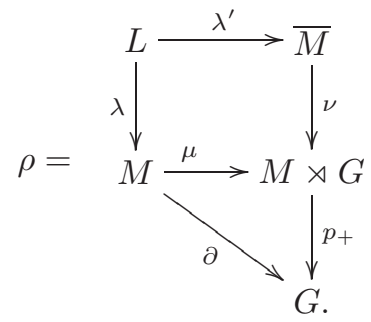

Therefore, $\mu: M \rightarrow M \rtimes G$ is the normal inclusion $m \mapsto\left(m, 1_{G}\right)$, and $\bar{M}$ is the normal subgroup of $M \rtimes G$ defined as $\bar{M}=\{\bar{m}\}_{m \in M}$, where, as before, $\bar{m}=\left(m, \partial(m)^{-1}\right)=$ $(m, 1)\left(1, \partial(m)^{-1}\right)$. Also, $p_{+}: M \rtimes G \rightarrow G$ is $p_{+}(m, w)=\partial(m) w$, thus $p_{+} \circ \nu=1_{G}$. Define a 2 -crossed module $\Psi(\rho)$ in the following way: As a group complex we put

$$
L \stackrel{\lambda}{\rightarrow} M \stackrel{\partial}{\rightarrow} G .
$$

(Note that $\partial=p_{+} \circ \mu$, thus given that $\nu \circ \lambda^{\prime}=\mu \circ \lambda$ it follows that $\partial \circ \lambda=1_{G}$.) The group $G$ acts on $L$ and $M$ through the inclusion $g \in G \mapsto(1, g) \in M \rtimes G$, as $g \triangleright m=$ ${ }^{g} m$ and $g \triangleright l={ }^{g} l$, where $g \in G, m \in M$ and $l \in L$, thus $\lambda$ and $\partial$ are equivariant under $G$. As the Peiffer lifting we put

$$
\{n, m\}=h\left(n m n^{-1}, \bar{n}^{-1}\right)=h\left(n m n^{-1},(1, \partial(n))\left(n^{-1}, 1\right)\right),
$$

which clearly is $G$-equivariant.

Lemma 2.5. The construction above defines a 2-crossed module and can be extended to a functor $\Psi$ from the category of neat crossed squares and neat maps into the category of 2-crossed modules, which extends in the obvious way to neat squared complexes, by sending the tail of a squared complex identically to the tail of the associated 2-crossed complex. 
Proof. We prove conditions 2 to 6 of the definition of a 2-crossed module. Note that

$$
\begin{aligned}
\lambda(\{n, m\}) & =\lambda\left(h\left(n m n^{-1}, \bar{n}^{-1}\right)\right)=\left(n m n^{-1}\right)^{\bar{n}^{-1}}\left(n m^{-1} n^{-1}\right) \\
& =\left(n m n^{-1}\right)^{\partial(n)} n^{-1}\left(n m^{-1} n^{-1}\right)=\left(n m n^{-1}\right)^{\partial(n)} m^{-1}=\langle n, m\rangle,
\end{aligned}
$$

and therefore condition 2 of the definition of a 2-crossed module follows. Also,

$$
\begin{aligned}
a \triangleright^{\prime} l & \doteq l\left\{\lambda\left(l^{-1}\right), a\right\}=l h\left(\lambda\left(l^{-1}\right) a \lambda(l),(\lambda(l), 1)\right)=\operatorname{lh}\left(\lambda\left(l^{-1}\right) a \lambda(l), \lambda^{\prime}(l)\right) \\
& =l\left({ }^{\left(l^{-1}\right) a \lambda(l)} l\right) l^{-1}={ }^{a} l, \text { where } a \in M \text { and } l \in L,
\end{aligned}
$$

which proves that $\left(\lambda: L \rightarrow M, \triangleright^{\prime}\right)$ is a crossed module. Given $l, k \in L$,

$$
\{\lambda(l), \lambda(k)\}=h\left(\lambda(l) \lambda(k) \lambda(l)^{-1},\left(\lambda\left(l^{-1}\right), 1\right)\right)=l k l^{-1} k^{-1}=[l, k] .
$$

To prove condition 5 , note that for $a, b, c \in M$ we have $\{a b, c\}=h\left(a b c b^{-1} a^{-1}, \overline{a b}^{-1}\right)$, whereas,

$$
\begin{aligned}
& \left\{a, b c b^{-1}\right\} \partial(a) \triangleright\{b, c\}=h\left(a b c b^{-1} a^{-1}, \bar{a}^{-1}\right) \partial(a) \triangleright h\left(b c b^{-1}, \bar{b}^{-1}\right) \\
& =h\left(a b c b^{-1} a^{-1}, \bar{a}^{-1}\right)^{\bar{a}^{-1}}\left({ }^{a} h\left(b c b^{-1}, \bar{b}^{-1}\right)\right)=h\left(a b c b^{-1} a^{-1}, \bar{a}^{-1}\right)^{\bar{a}^{-1}} h\left(a b c b^{-1} a^{-1},{ }^{a} \bar{b}^{-1}\right) \\
& =h\left(a b c b^{-1} a^{-1},\left(\bar{a}^{-1}\right)^{a}\left(\bar{b}^{-1}\right)\right)=h\left(a b c b^{-1} a^{-1}, \overline{a b}^{-1}\right) .
\end{aligned}
$$

Condition 4 is proved by noting that if $a, b, c \in M$, we have $\{a, b c\}=h\left(a b c a^{-1}, \bar{a}^{-1}\right)$, and also

$$
\{a, b\}(\partial(a) \triangleright b) \triangleright^{\prime}\{a, c\}=h\left(a b a^{-1}, \bar{a}^{-1}\right)^{\mu(\partial(a) \triangleright b)} h\left(a c a^{-1}, \bar{a}^{-1}\right) .
$$

Note that, since ${ }^{a} b=a b a^{-1}$, for each $a, b \in M$,

$$
\lambda\left(h\left(a b a^{-1}, \bar{a}^{-1}\right)\right)(\partial(a) \triangleright b)=\left(a b a^{-1}\right)\left(\bar{a}^{-1}\left(a b^{-1} a^{-1}\right)\right) \bar{a}^{-1}\left({ }^{a} b\right)=a b a^{-1} .
$$

Therefore, since $\left(\lambda: L \rightarrow M, \triangleright^{\prime}\right)$ is a crossed module,

$$
\{a, b\}(\partial(a) \triangleright b) \triangleright^{\prime}\{a, c\}=\left(\left(a b a^{-1}\right) h\left(a c a^{-1}, \bar{a}^{-1}\right)\right) h\left(a b a^{-1}, \bar{a}^{-1}\right)=h\left(a b c a^{-1}, \overline{a^{-1}}\right) .
$$

It now remains to prove condition 4 of the definition of a 2-crossed module. We have

$$
\begin{aligned}
\{\lambda(l), a\} & \{a, \lambda(l)\}=h\left(\lambda(l) a \lambda(l)^{-1}, \lambda^{\prime}\left(l^{-1}\right)\right) h\left(a \lambda(l) a^{-1}, \bar{a}^{-1}\right) \\
& =\left({ }^{\lambda(l) a \lambda(l)^{-1}} l^{-1}\right) l h\left(a \lambda(l) a^{-1}, \bar{a}^{-1}\right)=l\left({ }^{a} l^{-1}\right) h\left(a \lambda(l) a^{-1}, \bar{a}^{-1}\right) \\
& =l\left({ }^{a} l^{-1}\right) h\left(\lambda\left({ }^{a} l\right), \bar{a}^{-1}\right)=l^{\bar{a}^{-1}}\left({ }^{a} l^{-1}\right)=l \partial(a) \triangleright l^{-1} .
\end{aligned}
$$

The fact that $\Psi$ is a functor and that it can be extended to neat squared complexes is immediate from its construction, since we only consider neat 2-crossed module maps.

Theorem 2.6. The functor $\Psi$ is an equivalence of categories.

Proof. Given a 2-crossed module $(L \stackrel{\delta}{\rightarrow} E \stackrel{\partial}{\rightarrow} G,\{\},, \triangleright)$, we must check that (16) is a crossed square, where $h\left(m, \bar{n}^{-1}\right)=\left\{n, n^{-1} m n\right\}=\partial(n) \triangleright\left\{n^{-1}, m\right\}^{-1}$, equation (12), and also ${ }^{(m, g)} l=m \triangleright^{\prime}(g \triangleright l)$, which gives an action of $M \ltimes G$ on $L$ by automorphisms. We also put $\lambda^{\prime}(l)=\overline{\lambda(l)}=(\lambda(l), 1)$. 
That $\mu$ and $\nu$ define crossed modules follows from the fact that they are normal inclusions. That $\lambda^{\prime}$ and $\lambda$ define crossed modules follows from the fact that $\left(L \rightarrow M, \triangleright^{\prime}\right)$ is a crossed module. To prove that $h$ is $M \rtimes G$ equivariant, note that for each $g \in G$ we have $\left({ }^{g} m,{ }^{g} \bar{n}^{-1}\right)=h\left(g \triangleright m, \overline{g \triangleright n}^{-1}\right)=\left\{g \triangleright n, g \triangleright\left(n^{-1} m n\right)\right\}=g \triangleright$ $\left\{n, n^{-1} m n\right\}={ }^{g} h\left(m, \bar{n}^{-1}\right)$. Also, for each $a \in M$ we have

$$
\begin{aligned}
& \partial(n)^{-1} \triangleright h\left(^{a} m,{ }^{a} \bar{n}^{-1}\right)=\left\{\left(\partial(n)^{-1} \triangleright a\right) n^{-1} a^{-1}, a m a^{-1}\right\}^{-1} \\
& =\left(\left\{\left(\partial(n)^{-1} \triangleright a\right) n^{-1}, m\right\} \partial\left(n^{-1} a\right) \triangleright\left\{a^{-1}, a m a^{-1}\right\}\right)^{-1} \\
& \left.=\left(\left(\partial(n)^{-1} \triangleright a\right) \triangleright^{\prime}\left\{n^{-1}, m\right\}\right)\left\{\partial(n)^{-1} \triangleright a, \partial\left(n^{-1}\right) \triangleright m\right\} \partial\left(n^{-1} a\right) \triangleright\left\{a^{-1}, a m a^{-1}\right\}\right)^{-1} \\
& =\left(\left(\partial(n)^{-1} \triangleright a\right) \triangleright^{\prime}\left\{n^{-1}, m\right\}^{-1}\right)
\end{aligned}
$$

(we have used (12) and $\left\{\partial(n)^{-1} \triangleright a, \partial\left(n^{-1}\right) \triangleright m\right\}=\partial(n)^{-1} \triangleright\{a, m\}$ ), whereas,

$$
{ }^{a} h\left(m, \bar{n}^{-1}\right)=a \triangleright^{\prime}\left(\partial(n) \triangleright\left\{n^{-1}, m\right\}^{-1}\right)=\partial(n) \triangleright\left(\left(\partial(n)^{-1} \triangleright a\right) \triangleright^{\prime}\left\{n^{-1}, m\right\}^{-1}\right),
$$

where we have used (11). To prove equations (2) note that

$$
\begin{aligned}
& h\left(m m^{\prime}, \bar{n}^{-1}\right) \\
& =\partial(n) \triangleright\left\{n^{-1}, m m^{\prime}\right\}^{-1}=\partial(n) \triangleright\left(\left\{n^{-1}, m\right\}\left(\left(\partial\left(n^{-1}\right) \triangleright m\right) \triangleright^{\prime}\left\{n^{-1}, m^{\prime}\right\}\right)\right)^{-1} \\
& =\left(m \triangleright^{\prime} \partial(n) \triangleright\left\{n^{-1}, m^{\prime}\right\}\right)^{-1} \partial(n) \triangleright\left\{n^{-1}, m\right\}^{-1}=\left({ }^{m} h\left(m^{\prime}, \bar{n}^{-1}\right)\right) h\left(m, \bar{n}^{-1}\right)
\end{aligned}
$$

and

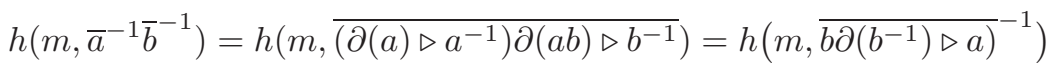

$$
\begin{aligned}
& =\partial(a b) \triangleright\left\{\left(\partial\left(b^{-1}\right) \triangleright a^{-1}\right) b^{-1}, m\right\}^{-1} \\
& =\partial(a b) \triangleright\left(\left(\left(\partial\left(b^{-1}\right) \triangleright a^{-1}\right) \triangleright^{\prime}\left\{b^{-1}, m\right\}\right)\left\{\partial\left(b^{-1}\right) \triangleright a^{-1}, \partial\left(b^{-1}\right) \triangleright m\right\}\right)^{-1} \\
& =\left(\partial(a) \triangleright\left\{a^{-1}, m\right\}\right)^{-1} \partial(a) \triangleright\left(a^{-1} \triangleright^{\prime} \partial(b) \triangleright\left\{b^{-1}, m\right\}\right)^{-1}=h\left(m, \bar{a}^{-1}\right)^{\bar{a}^{-1}} h\left(m, \bar{b}^{-1}\right) .
\end{aligned}
$$

Equations (3) can be proven as

$$
\lambda\left(h\left(m, \bar{n}^{-1}\right)\right)=\delta\left(\partial(n) \triangleright\left\{n^{-1}, m\right\}^{-1}\right)=m\left(\partial(n) \triangleright\left(n^{-1} m^{-1} n\right)\right)=m^{\bar{n}^{-1}} m^{-1},
$$

and also

$$
\lambda^{\prime}\left(h\left(m, \bar{n}^{-1}\right)\right)=\overline{\lambda\left(h\left(m, \bar{n}^{-1}\right)\right.}=\overline{m \partial(n) \triangleright\left(n^{-1} m^{-1} n\right)}=\left(m \partial(n) \triangleright\left(n^{-1} m^{-1} n\right), 1\right),
$$

whereas,

$\left({ }^{m} \bar{n}^{-1}\right) \bar{n}=(m, 1)\left(\partial(n) \triangleright n^{-1}, \partial(n)\right)\left(m^{-1}, 1\right)\left(n, \partial(n)^{-1}\right)=\left(m \partial(n) \triangleright\left(n^{-1} m^{-1} n\right), 1\right)$.

Equations (4) are easily proven.

\subsection{Totally free and 2-free 2-crossed modules and complexes (with or without a chosen basis)}

The reference now is [21]. Let $\partial: M \rightarrow G$ be a pre-crossed module. Let $\delta_{0}: K \rightarrow M$ be a set map such that $\operatorname{im}\left(\delta_{0}\right) \subset \operatorname{ker}(\partial)$. Then a 2-crossed module $\mathcal{H}=(F \stackrel{\delta}{\rightarrow} M \stackrel{\partial}{\rightarrow}$ $G, \triangleright,\{\}$,$) is said to be free on \delta_{0}: K \rightarrow M$ if there exists an inclusion $\iota: K \rightarrow F$ with $\delta_{0}=\delta \circ \iota$ such that the following universal property is satisfied: For any 2-crossed module $\mathcal{H}^{\prime}=\left(L \stackrel{\delta^{\prime}}{\rightarrow} E \stackrel{\partial}{\rightarrow} G, \triangleright,\{\},\right)$ and any map $\eta_{0}: K \rightarrow L$ such that $\delta^{\prime} \circ \eta_{0}=\delta_{0}$, 
there exists a unique map $\eta: F \rightarrow L$ extending $\eta_{0}$ and such that $\left(\eta\right.$,id,id): $\mathcal{H} \rightarrow \mathcal{H}^{\prime}$ is a 2 -crossed module morphism.

A free 2-crossed module on a map $\lambda_{0}: K \rightarrow \operatorname{ker}(\partial) \subset M$, where $\partial: M \rightarrow F$ is a pre-crossed module, is said to be totally free if $(M \rightarrow F)$ is itself a totally free precrossed module 1.1.1. This defines the category of totally free 2-crossed modules, a full subcategory of the category of 2-crossed modules. The category of totally free 2 -crossed modules with a chosen basis has objects given by $\left(S_{1}, S_{2}, K, \partial_{0}, \lambda_{0}\right)$, where $S_{1}, S_{2}$ and $K$ are sets, $\lambda_{0}: K \rightarrow \operatorname{ker}(\partial) \subset M$ is a map, where $\partial: M \rightarrow F$ is the free pre-crossed module on the map $\partial_{0}: S_{2} \rightarrow F$ and $F$ is the free group on $S_{1}$. The morphisms are given by all 2-crossed module maps between the associated totally free 2-crossed modules. A 2-crossed module $(L \rightarrow E \rightarrow G)$ is 2-free if the pre-crossed module $(E \rightarrow G)$ is totally free. The category of 2-free 2-crossed modules with chosen (pre-crossed module) basis is denoted by $\mathbf{2} \mathbf{X} \mathbf{m o d}_{\mathbf{b}}^{2-f r e e}$.

From the universal properties defining free crossed squares and free 2-crossed modules, and the construction of $\Psi$ follows:

Theorem 2.7. The functor $\Psi$ yields isomorphisms of categories between the categories of totally free or 2-free crossed squares with chosen basis and neat morphisms and the categories of totally free or 2-free 2-crossed modules with a chosen basis.

A 2-crossed complex (15) is totally free if the underlying 2-crossed module ( $L \rightarrow$ $E \rightarrow G)$ is totally free and, further, each $A_{n}$ is a free $\mathbb{Z}(G / \operatorname{im}(\partial))$-module. We denote the category of totally free 2 -crossed complexes with a chosen basis by $\mathbf{2} \mathbf{X} \mathbf{c o m p} \mathbf{p}$. There exists an isomorphism of categories $\Psi$ between the category of totally free squared complexes with a chosen basis 1.2 .5 and neat morphisms and the category of totally free 2 -crossed complexes with a chosen basis.

\subsection{Homotopy of pre-crossed module maps}

Let $(\partial: E \rightarrow G, \triangleright)$ and $\left(\partial: E^{\prime} \rightarrow G^{\prime}, \triangleright\right)$ be pre-crossed modules. Given a pre-crossed module map $\left(\psi: E \rightarrow E^{\prime}, \phi: G \rightarrow G^{\prime}\right)$, a $\phi$-derivation is a map $s: G \rightarrow E^{\prime}$ such that

$$
s(g h)=\left(\phi(h)^{-1} \triangleright s(g)\right) s(h), \text { for each } g, h \in G .
$$

Note that if $s$ is a derivation then $s\left(1_{G}\right)=1_{E}$, and also $s\left(g^{-1}\right)=\phi(g) \triangleright s(g)^{-1}$, if $g \in G$. Also, $s\left(g h g^{-1}\right)=\phi(g)^{-1} \triangleright\left(\left(\phi(h)^{-1} \triangleright s(g)\right) s(h) s(g)^{-1}\right)$. Let us define

$$
\begin{aligned}
\psi^{\prime \prime}(a) & =\psi(a)(s \circ \partial)(a), \text { where } a \in E, \\
\phi^{\prime}(g) & =\phi(g)(\partial \circ s)(g), \text { where } g \in G .
\end{aligned}
$$

Then $\phi^{\prime}: G \rightarrow G^{\prime}$ will be a group morphism, since given $g, h \in G$,

$$
\begin{aligned}
\phi^{\prime}(g h) & =\phi(h) \phi(g)(\partial \circ s)(g h)=\phi(g) \phi(h) \partial\left(\phi(h)^{-1} \triangleright s(g)\right) \partial(s(h)) \\
& =\phi(g)(\partial \circ s)(g))(\phi(h)(\partial \circ s)(h))=\phi^{\prime}(g) \phi^{\prime}(h) .
\end{aligned}
$$

However, given $a, b \in E$,

$$
\begin{aligned}
\psi^{\prime \prime}(a b) & =\psi(a b)(s \circ \partial)(a b)=\psi(a) \psi(b)\left(\phi\left(\partial(b)^{-1}\right) \triangleright s(\partial(a))\right) s(\partial(b)) \\
& =\psi^{\prime \prime}(a) s(\partial(a))^{-1} \psi(b)\left(\phi\left(\partial(b)^{-1}\right) \triangleright s(\partial(a))\right) s(\partial(b)) \\
& =\psi^{\prime \prime}(a)\left\langle\psi(b), \phi\left(\partial(b)^{-1}\right) \triangleright s(\partial(a))^{-1}\right\rangle^{-1} \psi(b) s(\partial(b)) \\
& =\psi^{\prime \prime}(a)\left\langle\psi(b), \phi\left(\partial(b)^{-1}\right) \triangleright s(\partial(a))^{-1}\right\rangle^{-1} \psi^{\prime \prime}(b) .
\end{aligned}
$$


Therefore $\psi^{\prime \prime}: E \rightarrow E^{\prime}$ is not a group morphism, in general, unless $\left(E^{\prime} \rightarrow G^{\prime}\right)$ is a crossed module. We therefore consider an additional map $t^{\prime}: E \rightarrow \operatorname{ker}(\partial) \subset E^{\prime}$ and put $\psi^{\prime}(a)=\psi^{\prime \prime}(a) t^{\prime}(a)$, for each $a \in E$. In order that $\left(\psi^{\prime}, \phi^{\prime}\right)$ is a pre-crossed module morphism, the map $t^{\prime}: E \rightarrow \operatorname{ker}(\partial) \subset E^{\prime}$ is to satisfy, for each $a, b \in E$ and $g \in G$,

$$
\begin{gathered}
t^{\prime}(a b)=\psi^{\prime \prime}(b)^{-1}\left\langle\psi(b), \phi\left(\partial(b)^{-1}\right) \triangleright s(\partial(a))^{-1}\right\rangle t^{\prime}(a) \psi^{\prime \prime}(b) t(b), \\
t^{\prime}(g \triangleright a)=s\left(g \partial(a) g^{-1}\right)^{-1}(\phi(g) \triangleright \psi(a))^{-1}\left(\phi^{\prime}(g) \triangleright \psi^{\prime}(a)\right) .
\end{gathered}
$$

We will then have

$$
\begin{aligned}
\psi^{\prime}(a b) & =\psi^{\prime \prime}(a)\left\langle\psi(b), \phi\left(\partial(b)^{-1}\right) \triangleright s(\partial(a))^{-1}\right\rangle^{-1} \psi^{\prime \prime}(b) t^{\prime}(a b) \\
& =\psi^{\prime \prime}(a) t^{\prime}(a) \psi^{\prime \prime}(b) t^{\prime}(b)=\psi^{\prime}(a) \psi^{\prime}(b)
\end{aligned}
$$

and also

$$
\begin{aligned}
\psi^{\prime}(g \triangleright a) & =\psi(g \triangleright a) s(\partial(t \triangleright a)) t^{\prime}(g \triangleright a) \\
& =(\phi(g) \triangleright \psi(a)) s\left(g \partial(a) g^{-1}\right) t^{\prime}(g \triangleright a)=\phi^{\prime}(g) \triangleright \psi^{\prime}(a) .
\end{aligned}
$$

Since $t^{\prime}: E \rightarrow E^{\prime}$ has values in $\operatorname{ker}(\partial)$ we have $\left(\partial \circ \psi^{\prime}\right)(a)=\left(\phi^{\prime} \circ \partial\right)(a)$. The pair $\left(\psi^{\prime}, \phi^{\prime}\right)$ therefore defines a pre-crossed module map $(E \rightarrow G) \rightarrow\left(E^{\prime} \rightarrow G^{\prime}\right)$.

We can express (19) in a different way; this will motivate the construction in 2.4.1:

$$
\begin{aligned}
& \phi(g)^{-1} \triangleright\left(t^{\prime}(g \triangleright a)\right) \\
& =s(g) s(\partial(a))^{-1}\left(\phi(\partial(a))^{-1} \triangleright s(g)^{-1}\right) \psi(a)^{-1}(\partial \circ s)(g) \triangleright\left(\psi(a) s(\partial(a)) t^{\prime}(a)\right) \\
& =s(g) s(\partial(a))^{-1}\left\langle\psi(a)^{-1}, s(g)^{-1}\right\rangle^{-1} \psi(a)^{-1} s(g)^{-1}(\partial \circ s)(g) \triangleright\left(\psi(a) s(\partial(a)) t^{\prime}(a)\right) .
\end{aligned}
$$

Thus, for each $a \in E$ and $g \in G$, we should have

$$
\begin{aligned}
t^{\prime}(g \triangleright a)=\phi(g) \triangleright\left(s(g) s(\partial(a))^{-1}\left\langle\psi(a)^{-1}, s(g)^{-1}\right\rangle^{-1} s(\partial(a)) s(g)^{-1}\right) \\
\phi(g) \triangleright\left\langle s(g), s(\partial(a))^{-1} \psi(a)^{-1}\right\rangle(\phi(g)(\partial \circ s)(g)) \triangleright t^{\prime}(a) .
\end{aligned}
$$

Definition 2.8 (Homotopy of pre-crossed module maps). Two pre-crossed module maps $(\psi, \phi)$ and $\left(\psi^{\prime}, \phi^{\prime}\right)$, from $(\partial: E \rightarrow G, \triangleright)$ to $\left(\partial: E^{\prime} \rightarrow G^{\prime}, \triangleright\right)$, are said to be homotopic if there exists a $\phi$-derivation $s: G \rightarrow E^{\prime}$, see equation (17), and a map $t^{\prime}: E \rightarrow \operatorname{ker}(\partial) \subset E^{\prime}$, satisfying equations (18) and (20), such that, for each $g \in G$ and $e \in E$, we have $\phi^{\prime}(g)=\phi(g) \partial(s(g))$ and also $\psi^{\prime}(e)=\psi(e)(s \circ \partial)(e) t^{\prime}(e)$.

\subsubsection{An auxiliary pre-crossed module}

Let $\mathcal{G}=(L \stackrel{\delta}{\rightarrow} E \stackrel{\partial}{\rightarrow} G, \triangleright,\{\}$,$) be a 2-crossed module. Equations (17), (18) and (20)$ can be nicely interpreted, and extended, by embedding them in the definition of a pre-crossed module $\left(E \ltimes_{*}\left(E \ltimes_{\triangleright^{\prime}} L\right) \stackrel{\beta}{\rightarrow} G \ltimes_{\triangleright} E, \bullet\right)$ associated with $\mathcal{G}$. This will play a major role in Subsections 2.5 and 2.6 .

We resume the notation of Subsection 2.1. Recall that $e \triangleright^{\prime} k \doteq k\left\{\delta(k)^{-1}, e\right\}$ defines a left action of $E$ on $L$ by automorphisms. Form the semidirect product $E \ltimes_{\triangleright^{\prime}} L$, see 
Remark 1.1. Consider the left action $*$ of $E$ on $E \ltimes_{\triangleright^{\prime}} L$, with (cf. equation (18)):

$$
\begin{aligned}
b *(e, k) & =\left(\partial(b) \triangleright e,\left(b \triangleright^{\prime}\left\{b^{-1}, \partial(b) \triangleright e^{-1}\right\}\right) b \triangleright^{\prime} k\right) \\
& =\left(\partial(b) \triangleright e,\left(\left\{b, e^{-1}\right\}^{-1}\right) b \triangleright^{\prime} k\right), \text { where } e, b \in E \text { and } k \in L ;
\end{aligned}
$$

see equation (13). To prove that this is a left action by automorphisms note that

$$
\begin{aligned}
& (b a) *(e, k)=\left(\partial(b) \partial(a) \triangleright e, b a \triangleright^{\prime}\left\{a^{-1} b^{-1}, \partial(b a) \triangleright e^{-1}\right\} b a \triangleright^{\prime} k\right) \\
& =\left(\partial(b) \partial(a) \triangleright e,\left(b \triangleright^{\prime}\left\{b^{-1}, \partial(b a) \triangleright e^{-1}\right\}\right)\left(b a \triangleright^{\prime}\left\{a^{-1}, \partial(a) \triangleright e^{-1}\right\}\right) b a \triangleright^{\prime} k\right) \\
& =b * a *(e, k),
\end{aligned}
$$

and also

$$
\begin{aligned}
& \left(b *\left(e^{-1}, k\right)\right)\left(b *\left(e^{-1}, k^{\prime}\right)\right) \\
& =\left(\partial(b) \triangleright e^{-1},\left(\{b, e\}^{-1}\right) b \triangleright^{\prime} k\right)\left(\partial(b) \triangleright e^{\prime-1},\left(\left\{b, e^{\prime}\right\}^{-1}\right) b \triangleright^{\prime} k^{\prime}\right) \\
& =\left(\partial(b) \triangleright\left(e^{\prime} e\right)^{-1},\left(\left(\partial(b) \triangleright e^{\prime}\right) \triangleright^{\prime}\{b, e\}^{-1}\right)\left(\left(\partial(b) \triangleright e^{\prime}\right) \triangleright^{\prime} b \triangleright^{\prime} k\right)\left(\left\{b, e^{\prime}\right\}^{-1}\right) b \triangleright^{\prime} k^{\prime}\right) \\
& =\left(\partial(b) \triangleright\left(e^{\prime} e\right)^{-1},\left(\left(\partial(b) \triangleright e^{\prime}\right) \triangleright^{\prime}\{b, e\}^{-1}\right)\left(\left\{b, e^{\prime}\right\}^{-1}\right)\left(b e^{\prime} \triangleright^{\prime} k\right) b \triangleright^{\prime} k^{\prime}\right) \\
& \left.=\left(\partial(b) \triangleright\left(e^{\prime} e\right)^{-1},\left\{b, e^{\prime} e\right\}^{-1}\right)\left(b e^{\prime-1} \triangleright^{\prime} k\right) b \triangleright^{\prime} k^{\prime}\right) \\
& =b *\left(\left(e^{\prime} e\right)^{-1},\left(e^{\prime-1} \triangleright^{\prime} k\right) k^{\prime}\right)=b *\left(\left(e^{-1}, k\right)\left(e^{\prime-1}, k^{\prime}\right)\right) .
\end{aligned}
$$

Consider the group $E \ltimes_{*}\left(E \ltimes_{\triangleright^{\prime}} L\right)$, whose group law is (Remark 1.1)

$$
(a, e, k)\left(a^{\prime}, e^{\prime}, k^{\prime}\right)=\left(a a^{\prime},\left(\partial\left(a^{\prime-1}\right) \triangleright e\right) e^{\prime},\left(\left(a^{\prime} e^{\prime}\right)^{-1} \triangleright^{\prime}\left(\left\{a^{\prime}, \partial\left(a^{\prime}\right)^{-1} \triangleright e^{-1}\right\} k\right)\right) k^{\prime}\right) .
$$

Therefore, if we put $a=(a, 1,1), e=(1, e, 1)$ and $k=(1,1, k)$, and the same for their images under $\triangleright$ and $\triangleright^{\prime}$, then

$$
(a, e, k)=a e k, \quad a k a^{-1}=a \triangleright^{\prime} k, \quad e k e^{-1}=e \triangleright^{\prime} k .
$$

Moreover, we have (23), (24) and (25), below:

$$
\begin{gathered}
a e a^{-1}=(\partial(a) \triangleright e)\left(a \triangleright^{\prime}\left\{a^{-1}, \partial(a) \triangleright e^{-1}\right\}\right)=(\partial(a) \triangleright e)\left(\left\{a, e^{-1}\right\}\right)^{-1}, \\
k a k^{-1}=a\left(a^{-1} \triangleright^{\prime} k\right) k^{-1} \text { and } e a e^{-1}=a\left(\partial\left(a^{-1}\right) \triangleright e\right)\left(\left\{a^{-1}, e^{-1}\right\}^{-1}\right) e^{-1}, \\
k l k^{-1}=\delta(k) \triangleright^{\prime} l=(\delta(k), 1,1) l(\delta(k), 1,1)^{-1}=(1, \delta(k), 1) l(1, \delta(k), 1)^{-1} .
\end{gathered}
$$

A particular case of the multiplication is

$$
(a, 1, k)\left(a^{\prime}, 1, k^{\prime}\right)=\left(a a^{\prime}, 1,\left(a^{\prime-1} \triangleright k\right) k^{\prime}\right), \text { where } a, a^{\prime} \in E \text { and } k, k^{\prime} \in L .
$$

Thus since $\left(\delta: L \rightarrow E, \triangleright^{\prime}\right)$ is a crossed module

$$
(\delta(l), 1, k)\left(\delta\left(l^{\prime}\right), 1, k^{\prime}\right)=\left(\delta(l) \delta\left(l^{\prime}\right), 1, l^{\prime-1} k l^{\prime} k^{\prime}\right), \text { where } k, k^{\prime}, l, l^{\prime} \in L .
$$

Lemma 2.9. There exists a left action $\bullet$ of $G \ltimes_{\triangleright} E$ on $E \ltimes_{*}\left(E \ltimes_{\triangleright^{\prime}} L\right)$, with

$$
\begin{aligned}
(g, x) \bullet(a, e, k)=(g \triangleright & a, g \triangleright\left(\left(\partial(a)^{-1} \triangleright x\right) e x^{-1}\right), \\
& \left.g \triangleright\left(x e^{-1} \triangleright^{\prime}\left\{a^{-1}, x^{-1}\right\}^{-1}\right) g \triangleright\left\{x, e^{-1} a^{-1}\right\}(g \partial(x)) \triangleright k\right),
\end{aligned}
$$


thus for each $g \in G$ and each $x \in E$ (this is to be compared with equation (20)):

$$
\begin{gathered}
g \bullet(a, e, k)=(g \triangleright a, g \triangleright e, g \triangleright k), \\
x \bullet(a, e, k)=\left(a,\left(\partial(a)^{-1} \triangleright x\right) e x^{-1}, x e^{-1} \triangleright^{\prime}\left\{a^{-1}, x^{-1}\right\}^{-1}\left\{x, e^{-1} a^{-1}\right\} \partial(x) \triangleright k\right) .
\end{gathered}
$$

A particular case which will be important later is

$$
x \bullet(\delta(k), 1, l)=\left(\delta(k), 1, k^{-1} \partial(x) \triangleright(k l)\right), \quad \text { where } x \in E \text { and } k, l \in L .
$$

Proof. First of all note that

$$
x \bullet g \bullet(a, e, k)=g \bullet g^{-1} \triangleright x \bullet(a, e, k) \text { and } g \bullet g^{\prime} \bullet(a, e, k)=\left(g g^{\prime}\right) \bullet(a, e, k) .
$$

The action by an element of $G$ is clearly by automorphisms. On the other hand,

$$
\left.(1, x, 1)(a, e, 1)\left(1, x^{-1}, 1\right)=\left(a,\left(\partial(a)^{-1} \triangleright x\right) e x^{-1},\left(x e^{-1} \triangleright^{\prime}\left\{a^{-1}, x^{-1}\right)\right\}^{-1}\right)\right),
$$

and therefore, putting $x=(1, x, 1)$, where $x \in E$,

$$
x \bullet(\text { aek })=\operatorname{xaex}^{-1}\left\{x, e^{-1} a^{-1}\right\} \partial(x) \triangleright k,
$$

from which it follows (note 22) and (25) and that $\left(\delta: L \rightarrow E, \triangleright^{\prime}\right)$ is a crossed module):

$$
\begin{aligned}
& (x \bullet(a e k))\left(x \bullet\left(a^{\prime} e^{\prime} k^{\prime}\right)\right) \\
& =x a e x^{-1}\left\{x, e^{-1} a^{-1}\right\}(\partial(x) \triangleright k)\left(x a^{\prime} e^{\prime} x^{-1}\right)\left\{x, e^{-1} a^{\prime-1}\right\} \partial(x) \triangleright k^{\prime} \\
& =x a e a^{\prime} e^{\prime} x^{-1}\left(\left(x e^{-1} a^{-1} x^{-1}\right) \triangleright^{\prime}\left(\left\{x, e^{-1} a^{-1}\right\} \partial(x) \triangleright k\right)\left\{x, e^{-1} a^{\prime-1}\right\} \partial(x) \triangleright k^{\prime}\right. \\
& =x a e a^{\prime} e^{\prime} x^{-1}\left\{x, e^{-1} a^{\prime-1}\right\}\left(\left(\partial(x) \triangleright\left(e^{\prime-1} a^{\prime-1}\right)\right) \triangleright^{\prime}\left(\left\{x, e^{-1} a^{-1}\right\} \partial(x) \triangleright k\right) \partial(x) \triangleright k^{\prime}\right. \\
& =x a e a^{\prime} e^{\prime} x^{-1}\left\{x, e^{-1} a^{\prime-1} e^{-1} a^{-1}\right\} \partial(x) \triangleright\left(\left(e^{\prime-1} a^{\prime-1}\right) \triangleright^{\prime} k\right) \partial(x) \triangleright k^{\prime} .
\end{aligned}
$$

On the other hand, by using (11), 21), (23) and (26)

$$
\begin{gathered}
x \bullet\left(a e k a^{\prime} e^{\prime} k^{\prime}\right)=x \bullet\left(a a^{\prime}\left(\partial\left(a^{\prime-1}\right) \triangleright e\right) e^{\prime}\left(\left(a^{\prime} e^{\prime}\right)^{-1} \triangleright^{\prime}\left(\left\{a^{\prime}, \partial\left(a^{\prime}\right)^{-1} \triangleright e^{-1}\right\} k\right) k^{\prime}\right) .\right. \\
=x a a^{\prime}\left(\partial\left(a^{\prime-1}\right) \triangleright e\right) e^{\prime} x^{-1}\left\{x, e^{\prime-1}\left(\partial\left(a^{\prime}\right)^{-1} \triangleright e^{-1}\right) a^{\prime-1} a^{-1}\right\} \\
\partial(x) \triangleright\left(\left(a^{\prime} e^{\prime}\right)^{-1} \triangleright^{\prime}\left(\left\{a^{\prime}, \partial\left(a^{\prime}\right)^{-1} \triangleright e^{-1}\right\} k\right) k^{\prime}\right) \\
=x a e\left(a^{\prime} \triangleright^{\prime}\left\{a^{\prime-1}, e\right\}\right) a^{\prime} e^{\prime} x^{-1}\left\{x, e^{\prime-1}\left(\partial\left(a^{\prime}\right)^{-1} \triangleright e^{-1}\right) a^{\prime-1} a^{-1}\right\} \\
\partial(x) \triangleright\left(\left(a^{\prime} e^{\prime}\right)^{-1} \triangleright^{\prime}\left(\left\{a^{\prime}, \partial\left(a^{\prime}\right)^{-1} \triangleright e^{-1}\right\} k\right) k^{\prime}\right) .
\end{gathered}
$$

Thus

$$
\begin{array}{r}
x \bullet\left(a e k a^{\prime} e^{\prime} k^{\prime}\right)=x a e a^{\prime} e^{\prime}\left(\left(x e^{-1}\right) \triangleright^{\prime}\left\{a^{\prime-1}, e\right\}\right)\left\{x, e^{-1}\left(\partial\left(a^{\prime}\right)^{-1} \triangleright e^{-1}\right) a^{\prime-1} a^{-1}\right\} \\
\partial(x) \triangleright\left(\left(a^{\prime} e^{\prime}\right)^{-1} \triangleright^{\prime}\left(\left\{a^{\prime}, \partial\left(a^{\prime}\right)^{-1} \triangleright e^{-1}\right\} k\right) k^{\prime}\right) .
\end{array}
$$

To show that $a e k \mapsto x \bullet(a e k)$ is a group morphism, we need to prove that, by (13):

$$
\begin{aligned}
\left\{x, e^{\prime-1} a^{-1} e^{-1} a^{-1}\right\} & =\left(x e^{\prime-1} \triangleright^{\prime}\left\{a^{\prime-1}, e^{-1}\right\}\right) \\
& \left\{x, e^{\prime-1}\left(\partial\left(a^{\prime}\right)^{-1} \triangleright e^{-1}\right) a^{\prime-1} a^{-1}\right\} \partial(x) \triangleright\left(e^{\prime-1} \triangleright^{\prime}\left\{a^{\prime-1}, e^{-1}\right\}\right)^{-1} .
\end{aligned}
$$


This follows from (note equation (14) and the fact that $\left(\delta: L \rightarrow E, \triangleright^{\prime}\right)$ is a crossed module):

$$
\begin{aligned}
& \left(x e^{\prime-1} \triangleright^{\prime}\left\{a^{\prime-1}, e^{-1}\right\}\right)\left\{x, e^{\prime-1}\left(\partial\left(a^{\prime}\right)^{-1} \triangleright e^{-1}\right) a^{\prime-1} a^{-1}\right\} \partial(x) \triangleright\left(e^{\prime-1} \triangleright^{\prime}\left\{a^{\prime-1}, e^{-1}\right\}^{-1}\right) \\
& =\left(\left(x e^{\prime-1}\right) \triangleright^{\prime}\left\{a^{\prime-1}, e^{-1}\right\}\right)\left\{x, e^{\prime-1}\left(\partial\left(a^{\prime}\right)^{-1} \triangleright e^{-1}\right) a^{\prime-1} a^{-1}\right\} \\
& \quad\left(x e^{\prime-1}\right) \triangleright^{\prime}\left(\left\{a^{\prime-1}, e^{-1}\right\}^{-1}\right)\left\{x, e^{-1}\left\langle a^{\prime-1}, e^{-1}\right\rangle e\right\} \\
& =\left(x e^{\prime-1}\left\langle a^{\prime-1}, e^{-1}\right\rangle e^{\prime} x^{-1}\right) \triangleright^{\prime}\left\{x, e^{\prime-1}\left(\partial\left(a^{\prime}\right)^{-1} \triangleright e^{-1}\right) a^{\prime-1} a^{-1}\right\}\left\{x, e^{\prime-1}\left\langle a^{\prime-1}, e^{-1}\right\rangle e^{\prime}\right\} \\
& =\left\{x, e^{\prime-1} a^{\prime-1} e^{-1} a^{-1}\right\} .
\end{aligned}
$$

We have used equation $(10)$. Let us now prove that $x \bullet y \bullet(a e k)=(x y) \bullet(a e k)$. First note that given $a, e, e^{\prime} \in E$, we have, by (10) and (22),

$$
\begin{aligned}
x \bullet\left(e a e^{\prime}\right) & =(x \bullet e)(x \bullet a)\left(x \bullet e^{\prime}\right)=x e^{-1}\left\{x, e^{-1}\right\} x a x^{-1}\left\{x, a^{-1}\right\} x e^{\prime} x^{-1}\left\{x, e^{\prime-1}\right\} \\
& =x e a x^{-1}\left(\left(x a^{-1} x^{-1}\right) \triangleright^{\prime}\left\{x, e^{-1}\right\}\right)\left\{x, a^{-1}\right\} x e^{\prime} x^{-1}\left\{x, e^{\prime-1}\right\} \\
& =x e a x^{-1}\left\{x, a^{-1} e^{-1}\right\} x e^{\prime} x^{-1}\left\{x, e^{-1}\right\}=x e a e^{\prime} x^{-1}\left\{x, e^{-1} a^{-1} e^{-1}\right\} .
\end{aligned}
$$

By using (26), we have $(x y) \bullet(a e k)=x y a e(x y)^{-1}\left\{x y, e^{-1} a^{-1}\right\} \partial(x y) \triangleright k$, whereas,

$$
\begin{aligned}
x \bullet y \bullet(a e k) & =(x \bullet y \bullet(a e))(x \bullet y \bullet k)=\left(x \bullet\left(y a e y^{-1}\left\{y, e^{-1} a^{-1}\right\}\right)\right)(\partial(x y) \triangleright k) \\
& =\left(x \bullet\left(y_{a e y}-1\right)\right)\left(x \bullet\left\{y, e^{-1} a^{-1}\right\}\right)(\partial(x y) \triangleright k) \\
& =x y a e y^{-1} x^{-1}\left\{x, y^{-1} e^{-1} a^{-1} y\right\}\left(\partial(x) \triangleright\left\{y, e^{-1} a^{-1}\right\}\right)(\partial(x y) \triangleright k) \\
& =x y a e y^{-1} x^{-1}\left\{x y, e^{-1} a^{-1}\right\}(\partial(x y) \triangleright k) .
\end{aligned}
$$

We therefore have a pre-crossed module $\left(E \ltimes_{*}\left(E \ltimes_{\triangleright^{\prime}} L\right) \stackrel{\beta}{\rightarrow} G \ltimes_{\triangleright} E\right.$, $\bullet$ ), where $\beta(a, e, k)=(\partial(a), e)$. Using (22) and $a \triangleright^{\prime} k \doteq k\left\{\delta\left(k^{-1}\right), a\right\}$, we obtain

$$
\begin{aligned}
& \left\langle a, a^{\prime}\right\rangle=\left(\left\langle a, a^{\prime}\right\rangle, 1,1\right) \quad\left\langle a, e^{\prime}\right\rangle=\left(1,1,\left\{a, e^{\prime}\right\}\right) \quad\left\langle a, k^{\prime}\right\rangle=\left(1,1,\left\{a, \delta\left(k^{\prime}\right)\right\}\right) \\
& \left\langle e, a^{\prime}\right\rangle=\left(1,1,\left\{e, a^{\prime}\right\}\right) \quad\left\langle e, e^{\prime}\right\rangle=\left(1,1,\left\{e, e^{\prime}\right\}\right) \quad\left\langle e, k^{\prime}\right\rangle=\left(1,1,\left\{e, \delta\left(k^{\prime}\right)\right\}\right) \\
& \left\langle k, a^{\prime}\right\rangle=\left(1,1,\left\{\delta(k), a^{\prime}\right\}\right) \quad\left\langle k, e^{\prime}\right\rangle=\left(1,1,\left\{\delta(k), e^{\prime}\right\}\right) \quad\left\langle k, k^{\prime}\right\rangle=\left(1,1,\left[k, k^{\prime}\right]\right) .
\end{aligned}
$$

Put $q \triangleright_{\text {ad }} q^{\prime}=q q^{\prime} q^{-1}$. Using Example 2.3, we can explicitly compute the Peiffer pairing in $\left(E \ltimes_{*}\left(E \ltimes_{\triangleright^{\prime}} L\right) \stackrel{\beta}{\rightarrow} G \ltimes_{\triangleright} E, \bullet\right)$, which will be used below:

$$
\begin{aligned}
& \left\langle a e k, a^{\prime} e^{\prime} k^{\prime}\right\rangle=\left\langle a, e k a^{\prime} e^{\prime} k^{\prime-1} k^{-1} e^{-1}\right\rangle \beta(a) \bullet\left\langle e k, a^{\prime} e^{\prime} k^{\prime}\right\rangle \\
& =\left(a e k a^{-1}\right) \triangleright_{\mathrm{ad}}\left\langle a, a^{\prime} e^{\prime} k^{\prime-1} k^{-1} e^{-1}\right\rangle\langle a, e k\rangle \beta(a) \bullet\left\langle e k, a^{\prime} e^{\prime}\right\rangle \\
& =\left(a e k a^{\prime} a^{-1}\right) \triangleright_{\mathrm{ad}}\left\langle a, e^{\prime} k^{\prime-1} k^{-1} e^{-1}\right\rangle\left(a e k a^{-1}\right) \triangleright_{\mathrm{ad}}\left\langle a, a^{\prime}\right\rangle\langle a, e k\rangle \beta(a) \bullet\left\langle e k, a^{\prime} e^{\prime}\right\rangle \\
& =\left(1,1,\left(a e \delta(k) a^{\prime} a^{-1}\right) \triangleright^{\prime}\left\{a, e^{\prime} \delta\left(k^{\prime-1} k^{-1}\right) e^{-1}\right\}\right) \\
& \left(\left\langle a, a^{\prime}\right\rangle, 1,\left\{a, a^{\prime}\right\}^{-1}\left(\left(a e \delta(k) a^{-1}\right) \triangleright\left\{a, a^{\prime}\right\}\right)\right)(1,1,\{a, e k\})\left(1,1, \partial(a) \triangleright\left\{e k, a^{\prime} e^{\prime}\right\}\right) \\
& =\left(\left\langle a, a^{\prime}\right\rangle, 1,\left\{a, a^{\prime}\right\}^{-1}\left(a e \delta(k) a^{\prime} a^{-1}\right) \triangleright^{\prime}\left\{a, e^{\prime} \delta\left(k^{\prime-1} k^{-1}\right) e^{-1}\right\}\right) \\
& \quad\left(1,1,\left(\left(a e \delta(k) a^{-1}\right) \triangleright\left\{a, a^{\prime}\right\}\right), 1\right)(1,1,\{a, e k\})\left(1,1, \partial(a) \triangleright\left\{e k, a^{\prime} e^{\prime}\right\}\right) \\
& =\left(\left\langle a, a^{\prime}\right\rangle, 1,\left\{a, a^{\prime}\right\}^{-1}\left\{a e \delta(k), a^{\prime} e^{\prime} \delta\left(k^{\prime}\right)\right\}\right) .
\end{aligned}
$$




\subsection{Quadratic derivations and homotopy of 2-crossed module maps}

Let $\mathcal{A}=(L \stackrel{\delta}{\rightarrow} E \stackrel{\partial}{\rightarrow} G, \triangleright,\{\}$,$) and \mathcal{A}^{\prime}=\left(L^{\prime} \stackrel{\delta}{\rightarrow} E^{\prime} \stackrel{\partial}{\rightarrow} G^{\prime}, \triangleright,\{\},\right)$ be 2-crossed modules. Let also $f=(\eta, \psi, \phi): \mathcal{A} \rightarrow \mathcal{A}^{\prime}$ be a 2-crossed module morphism. A pair $(t, s)$ of maps $s: G \rightarrow E^{\prime}$ and $t: E \rightarrow L^{\prime}$, satisfying equations (28), (29) and (31) below, will be called a quadratic $f$-derivation (this is to be compared with (17), (18), (20) and [3, IV-4]):

$$
\begin{gathered}
s(g h)=\left(\phi(h)^{-1} \triangleright s(g)\right) s(h), \text { where } g, h \in G, \\
t(a b)=(\psi(b)(s \circ \partial)(b))^{-1} \triangleright^{\prime}\left(\left\{\psi(b), \phi\left(\partial(b)^{-1}\right) \triangleright s(\partial(a))^{-1}\right\} t(a)\right) t(b),
\end{gathered}
$$

where $a, b \in E$. Thus, since $\left(\delta: L^{\prime} \rightarrow E^{\prime}, \triangleright^{\prime}\right)$ is a crossed module, by equation (11),

$$
t(\delta(k) \delta(l))=\eta(l)^{-1} t(\delta(k)) \eta(l) t(\delta(l)), \text { where } k, l \in L,
$$

and also (for each $g \in G$ and $e \in E$ ),

$$
\begin{aligned}
t(g \triangleright a)=\phi(g) \triangleright\left(s(g) s(\partial(a))^{-1} \triangleright^{\prime}\left\{\psi(a)^{-1}, s(g)^{-1}\right\}^{-1}\right) & \\
\phi(g) & \triangleright\left\{s(g), s(\partial(a))^{-1} \psi(a)^{-1}\right\}(\phi(g)(\partial \circ s)(g)) \triangleright t(a) .
\end{aligned}
$$

Thus, by using (13) and condition 4 of Definition 2.1, it follows that for each $g \in G$ and $l \in L$ :

$$
t(g \triangleright \delta(l))=\phi(g) \triangleright\left(\eta(l)^{-1} s(g) \triangleright(\eta(l) t(\delta(l)))\right) .
$$

The following very important lemma has an immediate proof. The pre-crossed module $\left(E \ltimes_{*}\left(E \ltimes_{\triangleright^{\prime}} L\right) \stackrel{\beta}{\rightarrow} G \ltimes_{\triangleright} E, \bullet\right)$ was constructed in 2.4.1.

Lemma 2.10. The pair of maps $t: E \rightarrow L^{\prime}$ and $s: G \rightarrow E^{\prime}$ is a quadratic $f$-derivation if and only if $H=\left(i_{2}, i_{1}\right)$ is a pre-crossed module morphism from $(E \rightarrow G, \triangleright)$ to $\left(E \ltimes_{*}\left(E \ltimes_{\triangleright^{\prime}} L\right) \stackrel{\beta}{\rightarrow} G \ltimes_{\triangleright} E, \bullet\right)$, where

$$
\begin{aligned}
& a \in E \stackrel{i_{2}}{\mapsto}(\psi(a), s \circ \partial(a), t(a)) \in E \ltimes_{*}\left(E \ltimes_{\triangleright^{\prime}} L\right), \\
& g \in G \stackrel{i_{1}}{\mapsto}(\phi(g), s(g)) \in G \ltimes_{\triangleright} E .
\end{aligned}
$$

Therefore, compare with Lemma IV (4.6) of [3]:

Lemma 2.11. If $\mathcal{F}$ is a totally free (or 2-free) 2-crossed module on the sets $S_{1}, S_{2}$ and $S_{3}$, and if $f: \mathcal{F} \rightarrow \mathcal{A}$ is a 2-crossed module map, then a quadratic $f$-derivation can be specified, uniquely, by its value on the sets $S_{1}, S_{2}$, with no compatibility relations.

The following is a 2-crossed complex analogue of proposition IV (4.4) in [3].

Lemma 2.12. Given a quadratic $f$-derivation $(t, s)$ we have

$$
t(\langle a, b\rangle)=\{\psi(a), \psi(b)\}^{-1}\{\psi(a) s(\partial(a)) \delta(t(a)), \psi(b) s(\partial(b)) \delta(t(b))\} .
$$

Proof. Since $\left(i_{1}, i_{2}\right)$ is a pre-crossed module morphism it preserves Peiffer pairings. Now use (27). Note that $\partial(\{e, f\})=1$ if $e, f \in E$. 
Theorem 2.13. For a quadratic $f$-derivation $(s, t)$, the map $f^{\prime}=\left(\eta^{\prime}, \psi^{\prime}, \phi^{\prime}\right)$, where

$$
\begin{aligned}
\eta^{\prime}(l) & =\eta(l)(t \circ \delta)(l), \text { where } l \in L, \\
\psi^{\prime}(a) & =\psi(a)(s \circ \partial)(a)(\delta \circ t)(a), \text { where } a \in E, \\
\phi^{\prime}(g) & =\phi(g)(\partial \circ s)(g), \text { where } g \in G
\end{aligned}
$$

is a morphism of 2-crossed modules, in which case we put $\stackrel{(f, s, t)}{\longrightarrow} f^{\prime}$.

Proof. At the level of pre-crossed module maps, this follows from the discussion in 2.4 . That $\psi^{\prime}$ is a $G$-equivariant group morphism follows from (30) and (32). Finally, Peiffer liftings are preserved due to (33) and $\partial(\langle E, E\rangle))=1$.

Definition 2.14. We say that two 2-crossed module maps $f, f^{\prime}: \mathcal{A} \rightarrow \mathcal{A}^{\prime}$ are homotopic if there exists a quadratic $f$-derivation $(s, t)$ such that $f \stackrel{(f, s, t)}{\longrightarrow} f^{\prime}$.

Contrary to the crossed complex case $1 \mathbf{1 0}, 25]$, homotopy of 2-crossed module maps is not an equivalence relation. This is related to the fact [15] that the composition of two 1-tranfors between Gray categories maps need not be a 1-tranfor.

\subsection{Free homotopy of totally free 2-crossed complexes}

This notion of homotopy between 2-crossed complex maps is analogous to the case of homotopies of quadratic chain complexes presented in [3, III-2]. Let $\mathcal{A}=$ $\left\{A_{n}, n \in \mathbb{N}\right\}$ and $\mathcal{C}=\left\{C_{n}, n \in \mathbb{N}\right\}$ be 2 -crossed complexes. Let $f=\left(f_{n}\right)$ be a 2 -crossed complex map $\mathcal{C} \rightarrow \mathcal{A}$. A quadratic $f$-derivation is a sequence of maps $\hat{s}_{i}: C_{i} \rightarrow A_{i+1}$ such that $\left(\hat{s}_{2}, \hat{s}_{1}\right)$ is a quadratic $\left(f_{3}, f_{2}, f_{1}\right)$-derivation, of 2-crossed modules, and all the remaining maps are $C_{1}$-equivariant for $n=3$ and $C_{1} / \partial\left(C_{2}\right)$-equivariant if $n \geqslant 4$. Further

$$
\hat{s}_{3}(\{e, f\})=1_{A_{4}} \text { for each } e, f \in C_{2} .
$$

(This last condition seems to be missing in [3], however it is apparently indispensable in the 2-crossed complex case.) We say that two 2-crossed complex maps $f, f^{\prime}: \mathcal{C} \rightarrow \mathcal{A}$ are homotopic and put $f \stackrel{(f, \hat{s})}{\longrightarrow} f^{\prime}$, if there exists a quadratic $f$-derivation $\hat{s}$ such that $f_{1}^{\prime}(c)=f_{1}(c) \partial \circ s_{1}(c)$, where $c \in C_{1}$ and $f_{n}^{\prime}(c)=f_{n}(c)\left(s_{n-1} \circ \partial(c)\right)\left(\partial \circ s_{n}(c)\right)$, where $c \in C_{n}$. By (34), and the fact that $\partial_{4}\left(A_{4}\right) \subset \operatorname{ker}\left(\partial_{3}\right)$ is central in $A_{3}$, it follows that $f^{\prime}$ is always a 2-crossed complex morphism for each quadratic $f$-derivation.

As in the case of 2-crossed modules, homotopy of 2-crossed complex maps is not an equivalence relation in general. Let us consider [3] the case when $\mathcal{F}=\left\{F_{n}, n \in \mathbb{N}\right\}$ is a totally free 2-crossed complex, on the chosen basis $\left\{B_{n}, n \in \mathbb{N}\right\}$, Subsection 2.3 . Given arbitrary set maps $s_{n}: B_{n} \rightarrow A_{n+1}$ and a 2-crossed complex map $f: \mathcal{F} \rightarrow \mathcal{A}$ there exists a unique quadratic $f$-derivation $\hat{s}$ extending $s$. Equation (34) is used to prove uniqueness.

Definition 2.15 (Free homotopy). Let $\mathcal{F}=\left\{F_{n}, n \in \mathbb{N}\right\}$ be a totally free 2-crossed complex, on the chosen basis $\left\{B_{n}, n \in \mathbb{N}\right\}$. Let $\mathcal{A}=\left\{A_{n}, n \in \mathbb{N}\right\}$ be a 2-crossed complex. A free homotopy is, by definition, a series of set maps $s_{n}: B_{n} \rightarrow A_{n+1}$. Clearly the set of free homotopies forms a group under the pointwise product of maps.

Definition 2.16. Two 2-crossed complex maps $f, f^{\prime}: \mathcal{F} \rightarrow \mathcal{A}$ are free homotopic if there exists a free homotopy $s=\left\{s_{n}\right\}$ such that $f \stackrel{(f, \hat{s})}{\longrightarrow} f^{\prime}$, where $\hat{s}$ is the unique 
extension of $s$ to a quadratic $f$-derivation. Free homotopy is an equivalence relation. Moreover, 2-crossed complex maps $\mathcal{F} \rightarrow \mathcal{A}$ and their free homotopies form a groupoid.

Definition 2.17. If $\mathcal{F}=(L \rightarrow M \rightarrow G)$ is a 2-free 2-crossed module, with chosen (pre-crossed module) basis, consisting of the sets $S_{1} \subset G$ and $S_{2} \subset M$, and $\mathcal{A}=(P \rightarrow$ $Q \rightarrow R)$ is any 2-crossed module, then a free homotopy $s=\left(s_{1}, s_{2}\right)$ is, by definition, given by set maps $s_{1}: S_{1} \rightarrow Q$ and $s_{2}: S_{2} \rightarrow P$. This defines a groupoid of 2-crossed module maps $\mathcal{F} \rightarrow \mathcal{A}$ and their free homotopies.

The category of totally free 2-crossed complexes with a chosen basis and with morphisms 2-crossed complex maps up to free homotopy is denoted by $\mathbf{2 X} \mathbf{X o m p}_{\mathbf{b}}^{\mathbf{f}} / \cong$. The category of 2-free 2-crossed modules, with a chosen basis, with morphisms

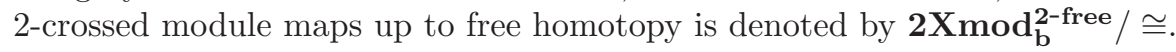

\section{2-crossed modules and pointed 3-types}

\subsection{The fundamental 2-crossed module $\Omega_{3}(X)$ of a CW-complex}

We now define the fundamental 2-crossed module $\Omega_{3}(X)$ of a canonical CWcomplex. Let $X$ be a canonical CW-complex 1.2.3. Therefore $X$ determines a pointed triad $X=\left(X ; X_{-}^{2}, X_{+}^{2}, *\right)$, with the 1 -skeleton $X^{1}$ of $X$ being a strong deformation retract of both $X_{-}^{2}$ and $X_{+}^{2}$. Let $p^{X}: X_{+}^{2} \rightarrow X^{1}$ be the obvious retraction. Note that $X^{1}$ is a subcomplex of the 1-dimensional CW-complex $X_{-}^{2} \cap X_{+}^{2}$. Consider the equivalence $\Psi$ between the categories of neat crossed squares and neat maps and of 2-crossed modules, Subsections 2.2 and 2.3, and also 1.2.1. Recall 1.2.3.

Definition 3.1. For a canonical CW-complex $X$, its fundamental 2-crossed module

$$
\Omega_{3}(X)=\left(\pi_{3}\left(X ; X_{-}^{2}, X_{+}^{2}\right) \stackrel{\delta}{\rightarrow} \pi_{2}\left(X_{-}^{2}, X_{-}^{2} \cap X_{+}^{2}\right) \stackrel{\partial}{\rightarrow} \pi_{1}\left(X_{+}^{2}\right) \cong \pi_{1}\left(X^{1}\right),\{,\}, \triangleright\right)
$$

is given by $\Omega_{3}(X)=\Psi\left(\rho_{3}(X)\right)$, where $\rho_{3}(X)$ is the fundamental crossed square of $X$ (which is neat in a natural way), 1.2.4 and 1.2.5. This defines a functor $\Omega_{3}$ from the category of canonical CW-complexes and bifiltered maps (or bifiltered homotopy classes of bifiltered maps) to the category of 2-crossed modules.

Let us be more specific about the construction of $\Omega_{3}(X)$. The map $\delta$ is the natural map appearing in the exact sequence of a triad; see 1.2.2. The map $\partial: \pi_{2}\left(X_{-}^{2}, X_{-}^{2} \cap\right.$ $\left.X_{+}^{2}\right) \rightarrow \pi_{1}\left(X_{+}^{2}\right)$ is the composition of the natural map $\pi_{2}\left(X_{-}^{2}, X_{-}^{2} \cap X_{+}^{2}\right) \rightarrow \pi_{1}\left(X_{-}^{2} \cap\right.$ $\left.X_{+}^{2}\right)$ with the map $p_{+}: \pi_{1}\left(X_{-}^{2} \cap X_{+}^{2}\right) \rightarrow \pi_{1}\left(X_{+}^{2}\right)$ induced by the inclusion $X_{-}^{2} \cap X_{+}^{2} \rightarrow$ $X_{+}^{2}$. It is easy to see that this defines a chain complex. The action of $\pi_{1}\left(X_{+}^{2}\right)$ on the rest of the complex $\Omega_{3}(X)$ is defined from the composition of the usual action of $\pi_{1}\left(X_{-}^{2} \cap\right.$ $\left.X_{+}^{2}\right)$ on $\pi_{3}\left(X ; X_{-}^{2}, X_{+}^{2}\right)$ and $\pi_{2}\left(X_{-}^{2}, X_{-}^{2} \cap X_{+}^{2}\right)$ with the map $\pi_{1}\left(X_{+}^{2}\right) \rightarrow \pi_{1}\left(X_{-}^{2} \cap X_{+}^{2}\right)$ induced by the retraction $p^{X}: X_{+}^{2} \rightarrow X^{1} \subset X_{-}^{2} \cap X_{+}^{2}$. Note that $\pi_{1}\left(X^{1}\right) \cong \pi_{1}\left(X_{+}^{2}\right)$. Given $m, n \in \pi_{2}\left(X_{-}^{2}, X_{-}^{2} \cap X_{+}^{2}\right)$, the Peiffer lifting is $\{n, m\}=h\left(n m n^{-1}, \bar{n}^{-1}\right)$, where $h: \pi_{2}\left(X_{-}^{2}, X_{-}^{2} \cap X_{+}^{2}\right) \times \pi_{2}\left(X_{+}^{2}, X_{-}^{2} \cap X_{+}^{2}\right) \rightarrow \pi_{3}\left(X ; X_{-}^{2}, X_{+}^{2}\right)$ is the generalised Whitehead product [1, 5. Recall Lemma 1.11.

Example 3.2. Consider the 2-sphere $S^{2}$ with a CW-decomposition with unique 0- and 2-cells. We have $\Omega_{3}\left(S^{2}\right)=(\mathbb{Z} \stackrel{n \mapsto 0}{\longrightarrow} \mathbb{Z} \rightarrow\{0\},\{\}$,$) with \{a, b\}=a b$. 
We can prove directly that $\Omega_{3}(X)$ is a 2 -crossed module. The advantage of defining it from Ellis' fundamental crossed square of a canonical CW-complex 17] is that the following non-trivial result follows directly from Brown-Loday's theorem [1, 12 (see 1.2.5) and Subsections 2.2 and 2.3.

Theorem 3.3. The fundamental 2 -crossed module of a 3 -dimensional canonical $\mathrm{CW}$ complex is the totally free 2-crossed module on the 1-, 2- and 3-cells of $X$ and their attaching maps.

Remark 3.4. The functor $\Omega_{3}$ is also defined for CW-complexes satisfying only conditions 1 and 2 of the definition of a canonical CW-complex. Lemma 1.10 tells us that it does not matter the way we transform a CW-complex $X$ in a homotopic canonical CW-complex $Y$ with $X^{2}=Y^{2}$ since in this case we will have $\Omega_{3}(X) \cong \Omega_{3}(Y)$.

\subsection{The fundamental 2-crossed complex $\Omega^{\infty}(X)$ of a CW-complex}

We have an isomorphism of categories $\Psi$ between the category of totally free squared complexes (with a chosen basis) and neat maps and the category $\mathbf{2} \mathbf{X} \mathbf{c o m p} \mathbf{p}_{\mathbf{b}}^{\mathbf{f}}$ of totally free 2-crossed complexes, with a chosen basis; see Subsections 2.2 and 2.3.

Definition 3.5. The fundamental 2-crossed complex of a canonical CW-complex $X$ is $\Omega^{\infty}(X)=\Psi\left(\rho^{\infty}(X)\right)$. Therefore the underlying group complex of $\Omega^{\infty}(X)$ is

$$
\rightarrow \pi_{5}\left(X^{5}, X^{4}\right) \rightarrow \pi_{4}\left(X^{4}, X^{3}\right) \rightarrow \pi_{3}\left(X^{3} ; X_{-}^{2}, X_{+}^{2}\right) \stackrel{\delta}{\rightarrow} \pi_{2}\left(X_{-}^{2}, X_{-}^{2} \cap X_{+}^{2}\right) \stackrel{\partial}{\rightarrow} \pi_{1}\left(X^{1}\right) .
$$

Theorem 3.6. The fundamental 2 -crossed complex of a canonical $\mathrm{CW}$-complex $X$ is the totally free 2-crossed complex on the cells of $X$ and their attaching maps.

We thus have functors $\Omega^{\infty}$ from the categories $\mathrm{CW}_{b}$ and $\mathrm{CW}_{b} / \cong$ of canonical CWcomplexes and bifiltered maps (up to bifiltered homotopy in the second case) into the category $\mathbf{2 X} \mathbf{X o m p} \mathbf{b}$ of totally free 2 -crossed complexes, with a chosen basis.

Remark 3.7. By the exact homotopy double complex of the triad $\left(X ; X_{-}^{2}, X_{+}^{2}\right)$, the homotopy groups $\pi_{1}(X), \pi_{2}(X)$ and $\pi_{3}(X)$ of a canonical CW-complex $X$ are the first three homology groups of $\Omega^{\infty}(X)$. The quadratic map $q: \pi_{2}(X) \rightarrow \pi_{3}(X)$ induced by the Hopf map $S^{3} \rightarrow S^{2}$ is encoded in the map $e \mapsto\{e, e\}$; see [17, page 104]. Note that for $e \in \operatorname{ker}(\partial)$ and $k \in \pi_{3}\left(X ; X_{-}^{2} \cap X_{+}^{2}\right)$ we have $\{e \delta(k), e \delta(k)\}=\{e, e\}$. For the remaining homology groups of $\Omega^{\infty}(X)$, see [17, Theorem 3].

Remark 3.8. The fundamental quadratic chain complex of a CW-complex [3] is a natural quotient of $\Omega^{\infty}(X)$; see [17]. This quotient [2] gives a reflection from the category of 2-crossed complexes into the category of quadratic chain complexes.

There exists an inclusion functor $\iota$ from the category of 2-crossed modules $\mathbf{2 X} \mathbf{m o d}$ into the category 2Xcomp of 2-crossed complexes, sending a 2-crossed module $\mathcal{G}=$ $(L \rightarrow E \rightarrow G)$ to the 2 -crossed complex $(\rightarrow\{0\} \rightarrow\{0\} \rightarrow L \rightarrow E \rightarrow G$ ) obtained by adding a trivial tail to $\mathcal{G}$. This inclusion functor has a left adjoint, the co-truncation functor $c \mathcal{T}$, sending a 2-crossed complex (15) to the 2-crossed module $\left(L / \partial\left(A_{4}\right) \rightarrow\right.$ $E \rightarrow G)$. The restriction $c \mathcal{T}: \mathbf{2} \mathbf{X} \mathbf{c o m p} \mathbf{p}_{\mathrm{b}}^{\mathrm{f}} \rightarrow \mathbf{2} \mathbf{X} \mathbf{m o d}_{\mathrm{b}}^{2-\text { free }}$ of the cotruncation functor descends to a functor $c \mathcal{T}: \mathbf{2 X} \operatorname{comp}_{\mathrm{b}}^{\mathrm{f}} / \cong \rightarrow \mathbf{2 X} \bmod _{\mathrm{b}}^{2 \text {-free }} / \cong$ since free homotopic 2crossed complex maps are sent to homotopic 2-crossed module maps under $c \mathcal{T}$; see Subsection 2.6. Lemma 3.9 follows immediately from one of the long exact sequences of the 4 -add (tetrad) $\left(X^{4} ; X^{3}, X_{-}^{2}, X_{+}^{2}\right)$; see [5, Section 8] and [18]. 
Lemma 3.9. Let $X$ be a canonical $\mathrm{CW}$-complex. Then $\Omega_{3}(X) \cong c \mathcal{T}\left(\Omega^{\infty}(X)\right)$.

\subsection{Realisation of 2-crossed complex maps}

The fact that there exists an isomorphism of categories $\Psi$ from the category of totally free squared complexes (with a chosen basis) and neat maps into the category of totally free 2-crossed complexes (with a chosen basis) permits us to use the results of 17] to prove the following result: Let $N \geqslant 4$ be an integer. A 2-crossed complex (15) is called $N$-truncated if $A_{n}$ is trivial for $n>N$. The following lemma follows from $1 \mathbf{1 7}$, Propositions 8 and 9]; see 1.2.6, namely Remark 1.17. It can be proven by an obvious induction on the $n$-skeleton of a CW-complex, as in the proof of Lemma 3.11.

Lemma 3.10 (Realisation-I). We have

1. Given a 5-truncated totally free 2-crossed complex $\mathcal{F}$, there exists a canonical CW-complex $X$ such that $\Omega^{\infty}(X)=\mathcal{F}$.

2. Given canonical $\mathrm{CW}$-complexes $X$ and $Y$, if $X$ is of dimension 4 , or smaller, then given any map $F: \Omega^{\infty}(X) \rightarrow \Omega^{\infty}(Y)$ there exists a bifiltered map $f: X \rightarrow$ $Y$ such that $\Omega^{\infty}(f)=F$, in which case we say that $f$ is a realisation of $F$. Given a subcomplex $X^{\prime}$ of $X$, any realisation $f^{\prime}: X^{\prime} \rightarrow Y$ of the restriction $F^{\prime}: \Omega^{\infty}\left(X^{\prime}\right) \rightarrow \Omega^{\infty}(Y)$ of $F$ to $\Omega\left(X^{\prime}\right)$, can be extended to a realisation of $F$.

If $X$ and $Y$ are 3-dimensional, then we can strengthen this result as:

Lemma 3.11 (Realisation-II). Let $X$ and $Y$ be canonical $\mathrm{CW}$-complexes of dimension 3 . The functor $\Omega_{3}$ yields a one-to-one correspondence between bifiltered homotopy classes of bifiltered maps $f: X \rightarrow Y$ and 2-crossed module maps $F: \Omega_{3}(X) \rightarrow \Omega_{3}(Y)$.

Proof. The discussion in 2.2 permits us to prove the analogous statement for the fundamental crossed square functor $\rho_{3}$, considering neat crossed square maps; see Remark 1.17. The fact that $\Omega_{3}$ is surjective on morphisms follows from Remark 1.17 .

We now prove that the assignment is injective. Let $W_{X}=X_{-}^{2} \cap X_{+}^{2}$ and $W_{Y}=$ $Y_{-}^{2} \cap Y_{+}^{2}$, both 1-dimensional CW-complexes. Suppose that $f$ and $f^{\prime}$ are bifiltered maps $X \rightarrow Y$ inducing the same (neat) map $F: \rho_{3}\left(X ; X_{-}^{2}, X_{+}^{2}\right) \rightarrow \rho_{3}\left(Y ; Y_{-}^{2}, Y_{+}^{2}\right)$ at the level of fundamental crossed squares. Since $\rho_{3}(f)$ and $\rho_{3}\left(f^{\prime}\right)$ define the same map $F: \pi_{1}\left(W_{X}\right) \rightarrow \pi_{1}\left(W_{Y}\right)$, there exists a pointed homotopy $H_{W}: W_{X} \times I \rightarrow W_{Y}$ connecting the restrictions of $f$ and $f^{\prime}$ to $W_{X}$. Since $\rho_{3}(f)$ and $\rho_{3}\left(f^{\prime}\right)$ are neat (that is $\left.F\left(\pi_{1}\left(X^{1}\right)\right) \subset \pi_{1}\left(Y^{1}\right)\right)$, we can suppose that $H_{W}\left(X^{1} \times I\right) \subset Y^{1}$. By using Theorem 1.4, together with the fact that $Y^{1}$ and $W_{Y}$ are aspherical, we can conclude that $H_{W}$ extends to a triad homotopy $H^{2}: X^{2} \times I \rightarrow Y^{2}$, connecting the restriction of $f$ and $f^{\prime}$ to $X^{2}$. By construction $H^{2}$ is bifiltered.

Let $R \subset X$ be made out of the interiors of the 3-cells of $X$. Consider a 3-cell $c$ of $X$, attaching along a map $t:\left(S^{2} ; S_{-}^{2}, S_{+}^{2}\right) \rightarrow\left(X^{2} ; X_{-}^{2}, X_{+}^{2}\right)$. The fact that $f(t)$ is triad homotopic to $f^{\prime}(t)$, for any 3 -cell, gives us a homotopy $T: R \times I \rightarrow Y$ connecting the restriction of $f$ and $f^{\prime}$ to $R$. A priori the extension of $T$ to the boundary of the 3-cells need not coincide with the previously defined homotopy $H^{2}: X^{2} \times I \rightarrow Y$. However, since this is a triad homotopy $H^{2}:\left(X^{2} \times I ; X_{-}^{2} \times I, X_{+}^{2} \times I\right) \rightarrow\left(Y^{2} ; Y_{-}^{2}, Y_{+}^{2}\right)$, with all spaces $Y_{-}^{2}, Y_{+}^{2}$ and $W_{Y}=Y_{-}^{2} \cap Y_{+}^{2}$ being aspherical, we can choose $T$ (by an obvious filling argument) so that it can be extended to the boundary of the 3-cells, coinciding with $H^{2}$ there. This finishes the proof. 
Let $X$ and $Y$ be canonical CW-complexes. Given a 2-crossed module morphism $F: \Omega_{3}(X) \rightarrow \Omega_{3}(Y)$, there exists a map $F^{\prime}: \Omega^{\infty}\left(X^{4}\right) \rightarrow \Omega^{\infty}(Y)$ such that $c \mathcal{T}\left(F^{\prime}\right)=F$ (see Lemma 3.9) since $\Omega^{\infty}\left(X^{4}\right)$ is a totally free 2-crossed complex. We have

Lemma 3.12. Let $X$ and $Y$ be canonical $\mathrm{CW}$-complexes with $Y$ a 3-type. Any 2crossed module map $F: \Omega_{3}(X) \rightarrow \Omega_{3}(Y)$ has a geometric realisation, and moreover this realisation $f$ is unique up to a triad homotopy $H:\left(X \times I ; X_{-}^{2} \times I, X_{+}^{2} \times I\right) \rightarrow$ $\left(Y ; Y_{-}^{2}, Y_{+}^{2}\right)$, satisfying moreover $H\left(X^{1} \times I\right) \subset Y^{1}$. If $X^{\prime}$ is a subcomplex of $X$ and if we already have a realisation $f^{\prime}$ of the restriction of $F$ to $\Omega_{3}\left(X^{\prime}\right)$, then we can suppose that $f$ extends $f^{\prime}$.

Proof. Choose a lifting $F^{\prime \prime}$ of $F$ to a map $F: \Omega^{\infty}\left(X^{4}\right) \rightarrow \Omega^{\infty}(Y)$. By using Lemma 3.10 we can geometrically realise $F^{\prime \prime}$ by a map $f^{4}: X^{4} \rightarrow Y$, which can certainly be extended to a map $f: X \rightarrow Y$, since $Y$ is a 3 -type. By Lemma 3.9 it follows that $\Omega_{3}(f)=F$. To prove unicity up to homotopy of the stated type, we can use the same method as in the previous lemma, taking into account the fact that $Y$ is a 3-type to extend a triad homotopy $X^{3} \times I \rightarrow Y$ to all of $X \times I$.

\subsection{Realisation of homotopies}

Let $X$ be a canonical CW-complex. Let $X \times^{\prime} I$ be the reduced cylinder of $X$, obtained from $X \times I$ by collapsing $X^{0} \times I$ to a point. Let $X_{0}=X \times\{0\}$ and $X_{1}=$ $X \times\{1\}$. The space $X \times^{\prime} I$ can be given the structure of a CW-complex with $X_{0}$ and $X_{1}$ embedded cellularly in $X \times^{\prime} I$, and intersecting along their common basepoint, the unique 0 -cell $X^{0}$ of $X$. We also have an extra $(i+1)$-cell $c^{\#}$ of $X \times^{\prime} I$ for each $i$-cell $c$ of $X$. Since we are mainly working with canonical CW-complexes, we discuss whether $X \times^{\prime} I$ is canonical or not; however see Remark 3.8 and Lemma 1.10. Given a cell $c$ of $X$, the element of $\Omega^{\infty}(X)$ yielded by $c$ will be denoted by $[c]$.

The 2-skeleton $\left(X \times^{\prime} I\right)^{2}$ of $X \times^{\prime} I$ is naturally a canonical CW-complex, where $X_{0}^{2}$ and $X_{1}^{2}$ are embedded cellularly. Any 1-cell $c^{1}$ of $X$ (with copies $c_{0}^{1}$ in $X_{0}$ and $c_{1}^{1}$ in $\left.X_{1}\right)$ gives a 2-cell $\left(c^{1}\right)^{\#}$ of $X \times^{\prime} I$, which, we impose, attaches along the concatenation $\left(\left[c_{0}^{1}\right]\right)^{-1}\left[c_{1}^{1}\right]$. In particular $\left[c_{1}^{1}\right]=\left[c_{0}^{1}\right] \partial\left(\left[\left(c^{1}\right)^{\#}\right]\right)$.

Each 2 -cell $c^{2}$ of $X$ (with copies $c_{0}^{2}$ in $X_{0}$ and $c_{1}^{2}$ in $\left.X_{1}\right)$ yields a 3 -cell $\left(c^{2}\right)^{\#}$ of $\left(X \times^{\prime}\right.$ $I)^{3}$, which however does not necessarily attach under a triad map $\left(S^{2} ; S_{-}^{2}, S_{+}^{2}\right) \rightarrow$ $\left(\left(X \times^{\prime} I\right)^{2} ;\left(X \times^{\prime} I\right)_{-}^{2},\left(X \times^{\prime} I\right)_{+}^{2}\right)$. Picking bifiltered approximations of the attaching maps of each 3 -cell $\left(c^{2}\right)^{\#}$, for a 2-cell $c^{2}$ of $X$, gives a canonical CW-complex $(X \square I)^{3}$ homotopic to $\left(X \times^{\prime} I\right)^{3}$. To be more specific, if $c^{2}$ attaches along the concatenation $\left[g_{1}\right]^{\theta_{1}} \cdots\left[g_{n}\right]^{\theta_{n}}$, where each $g_{i}$ is a 1-cell of $X$ and $\theta_{i} \in\{ \pm 1\}$, we make $\left(c^{2}\right)^{\#}$ attach along $\left(\left[c_{0}^{2}\right] s\left(\left[g_{1}\right]^{\theta_{1}} \cdots\left[g_{n}\right]^{\theta_{n}}\right)\right)^{-1}\left[c_{1}^{2}\right]$, which is a triad map, homotopic to the original attaching map (recall the proof of the bifiltered approximation theorem in 1.2.3). Here $s$ is the only derivation from the group $\pi_{1}\left(X^{1}\right)$ into $\pi_{2}\left(\left(X \times^{\prime} I\right)_{-}^{2},\left(X \times^{\prime} I\right)_{-}^{2} \cap\right.$ $\left.\left(X \times^{\prime} I\right)_{+}^{2}\right)$ such that for each 1-cell $g$ of $X$ we have that $[g] \mapsto\left[g^{\#}\right]$. We are considering the totally free pre-crossed module $\pi_{2}\left(\left(X \times^{\prime} I\right)_{-}^{2},\left(X \times^{\prime} I\right)_{-}^{2} \cap\left(X \times^{\prime} I\right)_{+}^{2}\right) \rightarrow$ $\pi_{1}\left(\left(X \times^{\prime} I\right)^{1}\right)$ and the inclusion map $\pi_{1}\left(X^{1}\right) \rightarrow \pi_{1}\left(\left(X \times^{\prime} I\right)^{1}\right)$. In particular, $\left[c_{1}^{2}\right]=$ $\left[c_{0}^{2}\right] s\left(\partial\left(c_{0}^{2}\right)\right) \delta\left(\left[\left(c^{2}\right)^{\#}\right]\right)$.

Similarly, if $c^{3}$ is a 3 -cell of $X$, then we have a 4 -cell $\left(c^{3}\right)^{\#}$ of $(X \square I)^{4}$, which we attach to $(X \square I)^{3}$ along $\left(\left[c_{0}^{3}\right] t\left(\delta\left(c^{3}\right)\right)\right)^{-1}\left[c_{3}^{1}\right]$, which is a map homotopic to the original attaching map of $\left(c^{3}\right)^{\#}$ in $\left(X \times^{\prime} I\right)^{3}$. Here $t: \pi_{2}\left(X_{-}^{2}, X_{-}^{2} \cap X_{+}^{2}\right) \rightarrow \pi_{3}\left(X \times^{\prime}\right.$ 
$\left.I ;\left(X \times^{\prime} I\right)_{-},\left(X \times^{\prime} I\right)_{+}\right)$is such that for each 2-cell $t\left(\left[c^{2}\right]\right)=\left[\left(c^{2}\right)^{\#}\right]$, and, moreover, $(t, s)$ is a quadratic derivation with respect to the inclusion $\Omega_{3}\left(X^{2}\right) \rightarrow \Omega_{3}\left(X^{2} \square I\right)$. In particular $\left[c_{1}^{3}\right]=\left[c_{0}^{3}\right] t\left(\partial\left(c_{0}^{3}\right)\right) \delta\left(\left[\left(c^{3}\right)^{\#}\right]\right)$.

Continuing this process yields a canonical CW-complex $X \square I$, homotopic to $X \times^{\prime}$ Iand whose bifiltered homotopy type is well defined by Lemma 1.10 .

Lemma 3.13. Let $X$ be a canonical CW-complex. There exists a canonical CWcomplex $X \square I$, containing $X_{0} \vee X_{1}$ as a subcomplex, such that there exist homotopy equivalences (which are the identity over $X_{0}=X \times\{0\}$ and $X_{1}=X \times\{1\}$ )

$$
r: X \square I \rightarrow X \times^{\prime} I \text { and } r^{\prime}: X \times^{\prime} I \rightarrow X \square I .
$$

Further, $X \square I$ has the same number of cells as $X \times^{\prime} I$ at each dimension.

The following lemma follows as a consequence of Lemma 3.13 .

Lemma 3.14. Let $X$ and $Y$ be canonical CW-complexes. Any two bifiltered maps $f_{0}, f_{1}: X \rightarrow Y$ are pointed homotopic if and only if there exists a bifiltered map $H^{\prime}: X \square I \rightarrow Y$ extending $f_{0} \vee f_{1}$.

Proof. If $H^{\prime}: X \square I \rightarrow Y$ extends $f_{0} \vee f_{1}$, then $H=H^{\prime} \circ r^{\prime}$ is a homotopy connecting $f_{0}$ and $f_{1}$. On the other hand, if $H: X \times^{\prime} I \rightarrow Y$ is a homotopy connecting $f_{0}$ and $f_{1}$ then we can take $H^{\prime}: X \square I \rightarrow Y$ to be a bifiltered approximation to $r \circ H$, coinciding with $f_{0} \vee f_{1}$ on $X_{0} \vee X_{1}$.

Lemma 3.15. Let $X$ be a canonical $\mathrm{CW}$-complex and $\mathcal{A}=\left(A_{n}\right)$ a 2-crossed complex. Consider two 2-crossed complex maps $F, F^{\prime}: \Omega^{\infty}(X) \rightarrow \mathcal{A}$. There exists a one-to-one correspondence between 2-crossed complex homotopies connecting $F$ and $F^{\prime}$ and 2crossed complex maps $H: \Omega^{\infty}(X \square I) \rightarrow \mathcal{A}$, extending $F \vee F^{\prime}: \Omega^{\infty}\left(X_{0} \vee X_{1}\right) \rightarrow \mathcal{A}$.

Proof. Consider the explicit construction of the CW-decomposition of $X \square I$. Since $\Omega^{\infty}(X \square I)$ is totally free, the set of 2-crossed complex maps $H: \Omega^{\infty}(X \square I) \rightarrow \mathcal{A}$ extending $F \vee F^{\prime}: \Omega^{\infty}\left(X_{0} \vee X_{1}\right) \rightarrow \mathcal{A}$ is given by the set of all assignments $\left(s_{i}\right)$ of an element of $A_{i+1}$ to each $i$-cell $c$ of $X$, satisfying the conditions motivated by the explicit form of the attaching maps of the $(i+1)$-cells $c^{\sharp}$ of $X \square I$. By construction it follows that we have a 2-crossed complex homotopy $F_{0} \stackrel{\left(F_{0}, \hat{s}\right)}{\longrightarrow} F_{1}$, where $\left(\hat{s}_{i}\right)$ is the unique extension of $\left(s_{i}\right)$ to an $F_{0}$-quadratic derivation. Clearly this correspondence is one-to-one.

Let $X$ be a canonical CW-complex and $\mathcal{G}$ a 2-crossed module. Consider two 2crossed module maps $F, F^{\prime}: \Omega_{3}(X) \rightarrow \mathcal{G}$. From the same argument there exists a oneto-one correspondence between 2-crossed module homotopies connecting $F$ and $F^{\prime}$ and 2-crossed module maps $H: \Omega_{3}(X \square I) \rightarrow \mathcal{A}$, extending $F \vee F^{\prime}: \Omega_{3}\left(X_{0} \vee X_{1}\right) \rightarrow$ $\mathcal{G}$.

Lemma 3.16. Let $X$ and $Y$ be canonical $\mathrm{CW}$-complexes with $Y$ and 3-type. Two bifiltered CW-complex maps $X \rightarrow Y$ are homotopic, as maps, if and only if their induced 2-crossed module maps are homotopic.

Proof. The proof follows from Lemmas 3.12, 3.14 and 3.15 . 


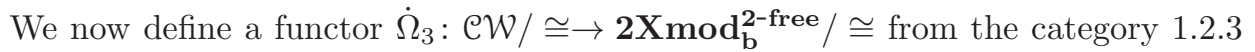
of canonical $\mathrm{CW}$-complexes and pointed homotopy classes of pointed maps into the category of 2-free 2-crossed modules (with a chosen basis) and homotopy classes of 2 -crossed module maps. On objects $\dot{\Omega}_{3}$ is defined in the same way as $\Omega_{3}$. If $f: X \rightarrow Y$ is a pointed map, then $\dot{\Omega}_{3}(f)$ is the equivalence class of $\Omega_{3}\left(f^{\prime}\right)$, where $f^{\prime}$ is a bifiltered approximation to $f$, Theorem 1.8. We have

Theorem 3.17. The restriction of $\dot{\Omega}_{3}$ to the category $\left\{3\right.$-types ${ }^{\text {can }}$ of canonical

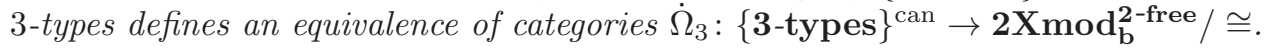

Proof. It is easy to see that given a 2-free 2-crossed module $\mathcal{G}=(L \rightarrow E \rightarrow G)$ there then exists a totally free 4-truncated 2-crossed complex $\mathcal{G}^{\prime}=\left(A \rightarrow L^{\prime} \rightarrow E \rightarrow\right.$ $G$ ) whose co-truncation is $\mathcal{G}$. Let $X^{\prime}$ be a 4-dimensional $\mathrm{CW}$-complex such that $\Omega^{\infty}\left(X^{\prime}\right) \cong \mathcal{G}^{\prime}$, thus $\Omega_{3}(X) \cong \mathcal{G}$, by Lemma 3.9. Let $X$ be a CW-complex obtained from $X^{\prime}$ by killing all of the homotopy groups $\pi_{i}$ of $X^{\prime}$ with $i \geqslant 4$ (the $4^{\text {th }}$-Postnikov section of $X)$. By the cellular approximation theorem, $\Omega_{3}(X) \cong \Omega_{3}\left(X^{\prime}\right) \cong \mathcal{G}$, thus $\dot{\Omega}_{3}$ is essentially surjective. That $\dot{\Omega}_{3}$ is fully faithful follows from the previous lemma.

\section{References}

[1] J.F. Adams, Algebraic topology - a student's guide, London Mathematical Society Lecture Note Series 4, Cambridge University Press, New York, 1972.

[2] Z. Arvasi and E. Ulualan, On algebraic models for homotopy 3-types, J. Homotopy Relat. Struct. 1 (2006), no. 1, 1-27

[3] H.J. Baues, Combinatorial homotopy and 4-dimensional complexes, With a preface by Ronald Brown, de Gruyter Expositions in Mathematics 2, Walter de Gruyter \& Co., Berlin, 1991.

[4] C. Berger, Double loop spaces, braided monoidal categories and algebraic 3type of space, in Higher homotopy structures in topology and mathematical physics (Poughkeepsie, NY, 1996), 49-66, Contemp. Math. 227, Amer. Math. Soc., Providence, RI, 1999.

[5] A.L. Blakers and W.S. Massey, The homotopy groups of a triad. I, Ann. of Math. (2) 53 (1951), 161-205.

[6] A.L. Blakers and W.S. Massey, Products in homotopy theory, Ann. of Math. (2) 58 (1953), 295-324.

[7] R. Brown, Computing homotopy types using crossed $n$-cubes of groups, Adams Memorial Symposium on Algebraic Topology 1 (Manchester, 1990), 187-210, London Math. Soc. Lecture Note Ser. 175, Cambridge Univ. Press, Cambridge, 1992.

[8] R. Brown, Topology and groupoids, BookSurge, LLC, Charleston, SC, 2006.

[9] R. Brown and N.D. Gilbert, Algebraic models of 3-types and automorphism structures for crossed modules, Proc. London Math. Soc. (3) 59 (1989), no. 1, $51-73$.

[10] R. Brown, P.J. Higgins and R. Sivera, Nonabelian algebraic topology, Filtered spaces, crossed complexes, cubical homotopy groupoids, EMS Tracts in Mathematics 15, European Mathematical Society, 2011. 
[11] R. Brown and J.L. Loday, Van Kampen theorems for diagrams of spaces. With an appendix by M. Zisman, Topology 26 (1987), no. 3, 311-335.

[12] R. Brown and J.L. Loday, Homotopical excision, and Hurewicz theorems for n-cubes of spaces, Proc. London Math. Soc. (3) 54 (1987), no. 1, 176-192.

[13] P. Carrasco and A.M. Cegarra, Group-theoretic algebraic models for homotopy types, J. Pure Appl. Algebra 75 (1991), no. 3, 195-235.

[14] D. Conduché, Modules croisés généralisés de longueur 2, Proceedings of the Luminy conference on algebraic $K$-theory (Luminy, 1983), J. Pure Appl. Algebra 34 (1984), no. 2-3, 155-178.

[15] S.E. Crans, A tensor product for Gray-categories, Theory Appl. Categ. 5 (1999), no. 2, 12-69 (electronic).

[16] S. Eilenberg and S. MacLane, Determination of the second homology and cohomology groups of a space by means of homotopy invariants, Proc. Nat. Acad. Sci. U.S.A. 32 (1946), 277-280.

[17] G. Ellis, Crossed squares and combinatorial homotopy, Math. Z. 214 (1993), no. 1, 93-110.

[18] G. Ellis and R. Steiner, Higher-dimensional crossed modules and the homotopy groups of $(n+1)$-ads, J. Pure Appl. Algebra 46 (1987), no. 2-3, 117-136.

[19] J. Faria Martins and R. Picken, The fundamental Gray 3-groupoid of a smooth manifold and local 3-dimensional holonomy based on a 2-crossed module, Differential Geometry and its Applications 29 (2011), no. 2, 179-206.

[20] J.L. Loday, Spaces with finitely many nontrivial homotopy groups, J. Pure Appl. Algebra 24 (1982), no. 2, 179-202.

[21] A. Mutlu and T. Porter, Freeness conditions for 2-crossed modules and complexes, Theory Appl. Categ. 4 (1998), no. 8, 174-194 (electronic).

[22] A. Mutlu and T. Porter, Freeness conditions for crossed squares and squared complexes, K-Theory 20 (2000), no. 4, 345-368.

[23] T. Porter, Crossed Menagerie: an introduction to crossed gadgetry and cohomology in algebra and topology, Online notes: http://ncatlab.org/ timporter/files/menagerie9.pdf

[24] J.H.C. Whitehead, On adding relations to homotopy groups, Ann. of Math. (2) 42 (1941), no. 2, 409-428.

[25] J.H.C. Whitehead, Combinatorial Homotopy. II, Bull. Amer. Math. Soc. 55 (1949), 453-496.

João Faria Martins jn.martins@fct.unl.pt

Departamento de Matemática, Faculdade de Ciências e Tecnologia, Universidade Nova de Lisboa, Quinta da Torre, 2829-516 Caparica, Portugal 